\title{
PREPRINT
}

Author-formatted, not peer-reviewed document posted on 07/03/2022

DOI: https://doi.org/10.3897/arphapreprints.e83016

\section{Identification key and checklist to the Swedish Phlaeothripidae (Thysanoptera)}

\author{
Emma Wahlberg, Carl-Axel Gertsson
}

\section{Disclaimer on biological nomenclature and use of preprints}

The preprints are preliminary versions of works accessible electronically in advance of publication of the final version. They are not issued for purposes of botanical, mycological or zoological nomenclature and are not effectively/validly published in the meaning of the Codes. Therefore, nomenclatural novelties (new names) or other nomenclatural acts (designations of type, choices of priority between names, choices between orthographic variants, or choices of gender of names) should NOT be posted in preprints. The following provisions in the Codes of Nomenclature define their status:

International Code of Nomenclature for algae, fungi, and plants (ICNafp)

Article 30.2: "An electronic publication is not effectively published if there is evidence within or associated with the publication that its content is merely preliminary and was, or is to be, replaced by content that the publisher considers final, in which case only the version with that final content is effectively published." In order to be validly published, a nomenclatural novelty must be effectively published (Art. 32.1(a)); in order to take effect, other nomenclatural acts must be effectively published (Art. 7.10, 11.5, 53.5, 61.3, and 62.3).

International Code of Zoological Nomenclature (ICZN)

Article: 21.8.3: "Some works are accessible online in preliminary versions before the publication date of the final version. Such advance electronic access does not advance the date of publication of a work, as preliminary versions are not published (Article 9.9)". 


\title{
Identification key and checklist to the Swedish Phlaeothripidae (Thysanoptera)
}

\author{
Emma Wahlberg ${ }^{1} \&$ Carl-Axel Gertsson ${ }^{2}$ \\ ${ }^{1}$ Department of Zoology, Swedish Museum of Natural History, P. O. Box 50007, SE-104 05 \\ Stockholm, Sweden. \\ ${ }^{2}$ Murarevägen 13, SE-227 30 Lund, Sweden.
}

Corresponding author: Emma Wahlberg, emma.wahlberg@nrm.se

\begin{abstract}
The Swedish fauna of thrips (Thysanoptera) in the family Phlaeothripidae consists of 49 species. A key to species found in Sweden is provided. One species is recorded as new for the country, and 10 new regional records are presented. A checklist of all Swedish species with regional distribution is also given.
\end{abstract}

\section{Keywords}

Thrips, identification, morphology, distribution, taxonomy, Tubulifera.

\section{Introduction}

The order Thysanoptera Haliday, 1836 are more commonly known as thrips, and are minute insects often not larger than $3 \mathrm{~mm}$. The larger species may reach $5 \mathrm{~mm}$ in size. Some has caught the attention not only from researchers but also from the commercial and private sector, due to some species that are considered as pests in agriculture (Paine 1992) and even as invasive (Held et al. 2003; Boyd \& Held 2006). The least studied group in Sweden is the family Phlaeothripidae Uzel, 1895. Some species are found in flowers, e.g. in the genus Haplothrips Amyot \& Serville, 1843 (Fig. 1), but most of the known species in Sweden are found in soil, leaf litter and decaying wood. The small size and secluded lifestyle might be reasons for the relatively few taxonomic studies of the order in general and this family in specific.

The research regarding Palaearctic taxa is scarce. Only a few regional checklists have been published in recent years, and the most relevant identification keys are focused on Great Britain (Mound et al. 1976; Kirk 1996). In recent years the fauna of Poland has been studied more extensively, a region with a previously similarly understudied thrips fauna, which has led to a large gain in both taxonomic and ecological knowledge (Kucharczyk and Zawirska 1994; Kąkol and Kucharczyk 2003; Kucharczyk 2004; Kucharczyk and Kucharczyk 2008; Dubovský et al. 2010). Most of the knowledge of Swedish taxa is based on older identification literature, e.g. Ahlberg (1926), Mound et al. (1976) and Kirk (1996), often not specific for Scandinavian conditions. A few papers have been published reporting new species at irregular intervals, reporting sporadic observations (Qvick 1977; Vasiliu-Oromulu et al. 2000; Kobro 2011; Sörensson 2012; Gertsson 2015a; Gertsson \& Fägerström 2017). Kobro \& Rafoss (2006) produced a key to the genus Hoplothrips in Phlaeothripidae. Kobro (2013) produced an identification key to Norwegian thrips in general, but only covered the most common and for amateurs easily distinguished species. The complete overlap of Swedish and Norwegian species is currently not investigated. Gertsson (2015b) provided a checklist of Nordic thrips. However, this was based only on previously collected material in museum 
collections. Recently new records to the fauna were made from freshly collected material, with a total of 5 new species for Sweden and several new regional records (Gertsson and Fägerström 2017; Gertsson 2021; Gertsson et al. 2022). In this paper we update the Swedish checklist of the family Phlaeothripidae and provide an identification key to the species with photographic illustrations.

\section{Material and methods}

For this study we have examined representative specimens from the collections of The Swedish Museum of Natural History, Sweden (NHRS), Lund Museum of Zoology (MZLU), Sweden, Forschungsinstitut und Naturmuseum Senckenberg (SMF), Germany and the private collections of Sverre Kobro and Manfred R. Ulitzka. In addition, freshly collected material have been used, prepared on slides with Euparal according to the method outlined in Kobro (2013). Exception for this method is preservation prior to maceration and the maceration step, where in this study fresh material has been stored in $80 \%$ ethanol prior to DNA extraction. Maceration has thereafter been carried out during DNA extraction before preparation on microscopic slides. This method has successfully been used for one step DNA extraction and maceration for small insect specimens (Wahlberg and Johanson 2018; Wahlberg 2019). DNA extract is stored at the NHRS for further studies. The material was examined and photographed using manual focus stacking on Nikon Eclipse 80i and Swift 380T microscopes, with Nikon DS-Fi1 and Swift SC1003 cameras. Photos were automatically aligned and stacked using Helicon Focus 8.0.4 and Swift Imaging 3.0, and edited and finalized in Adobe Photoshop CC 23.2.0. The distributional data are provided on county level. The material collected and preserved during this project is deposited at the NHRS.

\section{Swedish faunistic provinces and abbreviations}

Sweden is traditionally divided in to faunistic provinces, most based on historical cultural regions overlapping with administrative counties (Fig. 2). They are in the checklist and map abbreviated as below, from south to north.

$$
\begin{aligned}
& \text { Sk }=\text { Skåne } \\
& \mathrm{Bl}=\text { Blekinge } \\
& \mathrm{Ha}=\text { Halland } \\
& \mathrm{Sm}=\text { Småland } \\
& \text { Öl }=\text { Öland } \\
& \mathrm{Go}=\text { Gotland } \\
& \mathrm{GS}=\text { Gotska Sandön } \\
& \text { Ög }=\text { Östergötland } \\
& \mathrm{Vg}=\text { Västergötland } \\
& \mathrm{Bo}=\text { Bohuslän } \\
& \mathrm{Ds}=\text { Dalsland } \\
& \mathrm{Nä}=\text { Närke } \\
& \mathrm{Sö}=\text { Södermanland } \\
& \mathrm{Up}=\text { Uppland } \\
& \mathrm{Vs}=\text { Västmanland } \\
& \mathrm{Vr}=\text { Värmland } \\
& \mathrm{Dr}=\text { Dalarna } \\
& \mathrm{Gä}=\text { Gästrikland } \\
& \mathrm{Hs}=\text { Hälsingland }
\end{aligned}
$$




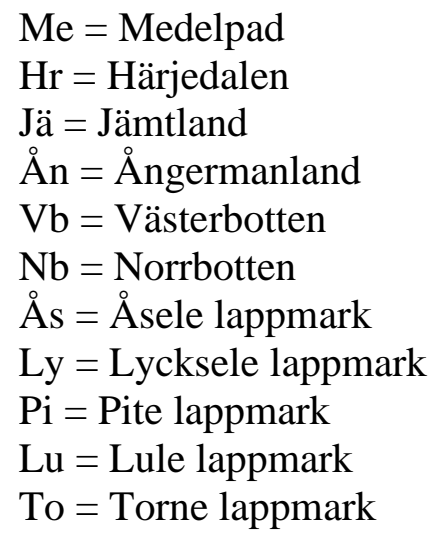

\section{Characters}

The identification key is intended to be used for adult specimens, both females and males in various life stages and both winged and apterous forms. For this reason, some species that express great intraspecic variation it is possible to key out one species at several locations in the key. In Thysanoptera the most important morphological characters for species identification include antennal shape, presence, shape, and length of setae, feeding structures, and measurements of segments (Fig. 3). This always requires high magnification and specimen preparation. Large setae may be blunt, expanded (Fig. 10G), or acute at apex, and care need to be taken in preparation for avoiding collapse of expanded apices. The antennal segments often carry sensory organs in the shape of large trichomes. These are more robust and broader than bristles that they might be confused with (Fig. 6A-C). Maxillary stylets are parts of the feeding apparatus and can be seen in macerated specimens (Fig. 4A-B), and the width of the stylets and presence or absence of the median extension called maxillary bridge are used for separation of subfamilies and species groups. The last segment, segment $X$, of the abdomen may be either tapering and longitudinally divided or complete and tube shaped. The latter being one of the defining characters of the family Phlaeothripidae (Figs 3).

Comprehensive and detailed descriptions of the anatomy and morphology of Thysanoptera are provided in Schliephake and Klimt (1979) and Moritz (2006).

\section{Taxonomy}

\section{Phlaeothripidae Uzel, 1895}

\section{Diagnosis}

The last abdominal segment, segment $\mathrm{X}$, tubular in both males and females (Figs 3, 5C, 7AB), without longitudinal division and without saw-like ovipositor. If wings present, then forewings without longitudinal veins and surface without microtrichia (Fig. 3).

\section{Notes}

There are about 3700 known species of Phlaeothripidae in the world (Mound and Tree 2021). Most of these species are described from tropical and sub-tropical areas. In Sweden 49 species are known. The Phlaeothripidae are diverse in their biology; living of decaying matter, pollen, fungal spores and hyphae and prey, and sometimes expressing polymorphism and sociality (Kirk 1996; Mound 2004).

\section{Key to the species of Phlaeothripidae from Sweden}




\begin{tabular}{|c|c|c|}
\hline \multirow[t]{2}{*}{1.} & $\begin{array}{l}\text { Maxillary stylets broad, at least } 5 \mu \mathrm{m} \text { wide } \\
\text { (twice as wide as base of postocular setae) } \\
\text { (Fig. 4A). }\end{array}$ & Idolothripinae 1 \\
\hline & $\begin{array}{l}\text { Maxillary stylets slender, less than } 5 \mu \mathrm{m} \text { wide } \\
\text { (Fig. 4B). }\end{array}$ & Phlaeothripinae 7 \\
\hline & Idolothripinae Bagnall, 1908 & \\
\hline \multirow[t]{2}{*}{$2(1)$. } & $\begin{array}{l}\text { Anterior margin of ocellar triangle with long } \\
\text { setae (Fig. 4C); large and dark species with } \\
\text { elongated head. }\end{array}$ & 3 \\
\hline & $\begin{array}{l}\text { Setae at anterior margin of ocellar triangle } \\
\text { short or absent (Fig. 4D). }\end{array}$ & 5 \\
\hline \multirow[t]{2}{*}{$3(2)$. } & Lateral wings of pelta slender (Fig. 5E). & Megalothrips bonanni \\
\hline & $\begin{array}{l}\text { Lateral wings of pelta (abdominal tergite I) } \\
\text { triangular (Fig. 5D). }\end{array}$ & 4 \\
\hline \multirow[t]{2}{*}{$4(2)$. } & Tarsi pale and tibia brown (Fig. 5C). & Bacillothrips nobilis \\
\hline & Tarsi and tibia yellow (Fig. 5F). & Megathrips lativentris \\
\hline \multirow[t]{2}{*}{$5(2)}$. & Eyes ventrally elongated (Fig. 5A). & 6 \\
\hline & Eyes not ventrally elongated. & 7 \\
\hline \multirow[t]{2}{*}{$6(5)$} & $\begin{array}{l}\text { Body (excluding antennae, legs and wings) } \\
\text { brown with yellow pronotum and yellow } \\
\text { transverse band on metanotum. }\end{array}$ & Bolothrips bicolor \\
\hline & Body uniformly brown. & Bolothrips dentipes \\
\hline \multirow[t]{3}{*}{$7(5)$} & $\begin{array}{l}\text { Maxillary stylets close together, meeting or } \\
\text { almost meeting medially (Fig. 4D). }\end{array}$ & Cryptothrips nigripes \\
\hline & $\begin{array}{l}\text { Maxillary stylets widely separated, at least } \\
\text { half of the heads width apart (Fig. } 4 \text { A). }\end{array}$ & Bolothrips icarus \\
\hline & Phlaeothripinae Uzel, 1895 & \\
\hline \multirow[t]{2}{*}{$8(1)$. } & Maxillary bridge present (Fig. 5B). & 9 \\
\hline & Maxillary bridge absent (Fig. 4B). & 28 \\
\hline \multirow[t]{2}{*}{$9(8)$. } & $\begin{array}{l}\text { Antennal segment IV with } 2 \text { sense cones (Fig. } \\
6 \mathrm{~A} \text { ). }\end{array}$ & Xylaplothrips fuliginosus \\
\hline & $\begin{array}{l}\text { Antennal segment IV with } 3-4 \text { sense cones } \\
\text { (Fig. } 6 \mathrm{~B}-\mathrm{C} \text { ). }\end{array}$ & Haplothrips $\mathbf{1 0}$ \\
\hline \multirow[t]{2}{*}{$10(9)$. } & $\begin{array}{l}\text { Antennal segment III with no sense cones (cf. } \\
\text { Fig. 9E). }\end{array}$ & Haplothrips minutus \\
\hline & $\begin{array}{l}\text { Antennal segment III with at least } 1 \text { sense } \\
\text { cone. }\end{array}$ & 11 \\
\hline \multirow[t]{2}{*}{$11(10)$. } & Antennal segment III with 1 sense cone. & 12 \\
\hline & Antennal segment III with 2 sense cones. & 14 \\
\hline \multirow[t]{2}{*}{$12(11)$. } & $\begin{array}{l}\text { Postocular setae expanded or bluntly pointed } \\
\text { (Fig. 6D). }\end{array}$ & Haplothrips subtilissimus \\
\hline & Postocular setae acute (Fig. 6E). & 13 \\
\hline \multirow[t]{2}{*}{$13(12)$} & $\begin{array}{l}\text { Tube more than } 2.3 \text { times longer than wide } \\
\text { (Fig. 7A). }\end{array}$ & Haplothrips alpester \\
\hline & $\begin{array}{l}\text { Tube less than } 2.3 \text { times longer than wide } \\
\text { (Fig. 7B). }\end{array}$ & Haplothrips aculeatus \\
\hline
\end{tabular}




\begin{tabular}{|c|c|c|}
\hline \multirow[t]{2}{*}{$14(11)$} & $\begin{array}{l}\text { Postocular setae short, no longer than the } \\
\text { width of the eye (Fig. 6F). }\end{array}$ & 15 \\
\hline & $\begin{array}{l}\text { Postocular setae well developed and long } \\
\text { (Fig. 7C). }\end{array}$ & 18 \\
\hline \multirow[t]{2}{*}{15 (14). } & $\begin{array}{l}\text { Setae S1 on tergite IX blunt apically (Fig. } \\
\text { 7D). }\end{array}$ & 16 \\
\hline & Setae S1 on tergite IX acute. & 17 \\
\hline \multirow[t]{2}{*}{$16(15)$} & $\begin{array}{l}\text { Antennal segment IV yellow at base (Fig. } \\
\text { 7E). }\end{array}$ & Haplothrips leucanthemi \\
\hline & Antennal segment IV wholly brown (Fig. 7F). & Haplothrips propinquus \\
\hline \multirow[t]{2}{*}{$17(15)$} & $\begin{array}{l}\text { Both anteromarginal and anteroangular setae } \\
\text { stout and at least twice as long as discal setae } \\
\text { (Fig. } 7 \mathrm{G} \text { ). }\end{array}$ & Haplothrips alpicola \\
\hline & $\begin{array}{l}\text { Anteromarginal setae minute, anteroangular } \\
\text { setae sometimes longer both not as stout. }\end{array}$ & Haplothrips angusticornis \\
\hline \multirow[t]{2}{*}{$18(14)$} & $\begin{array}{l}\text { Setae S1 on tergite IX blunt apically (cf. Fig. } \\
\text { 7E). }\end{array}$ & 19 \\
\hline & Setae S1 on tergite IX acute. & 21 \\
\hline \multirow[t]{2}{*}{$19(18)$} & Postocular setae acute (Fig. 7H). & Haplothrips tritici \\
\hline & Postocular setae bluntly pointed (Fig. 5B). & 20 \\
\hline \multirow[t]{2}{*}{$20(19)$} & $\begin{array}{l}\text { Maxillary stylets about a third of head's width } \\
\text { apart (Fig. 5B); tibia I brown }\end{array}$ & Haplothrips senecionis \\
\hline & $\begin{array}{l}\text { Maxillary stylets less than a third of the } \\
\text { head's width apart (Fig. 7C); tibia I yellow } \\
\text { apically }\end{array}$ & Haplothrips statices \\
\hline \multirow[t]{2}{*}{21 (18). } & $\begin{array}{l}\text { Distal cilia of forewing with barbs (in high } \\
\text { magnification), in lower magnification visible } \\
\text { as a rough or frizzled surface (Fig. 8A). }\end{array}$ & Haplothrips setiger \\
\hline & Distal cilia of forewing smooth & 22 \\
\hline \multirow[t]{2}{*}{$22(21)$. } & Postocular setae bluntly pointed (Fig. 8B). & 23 \\
\hline & Postocular setae acute (Fig. 7H). & 24 \\
\hline \multirow[t]{2}{*}{$23(22)$} & $\begin{array}{l}\text { Maxillary stylets about a fourth of head's } \\
\text { width apart (Fig. 8B); tibia I yellow but } \\
\text { brown basally. }\end{array}$ & Haplothrips verbasci \\
\hline & $\begin{array}{l}\text { Maxillary stylets about half of head's width } \\
\text { apart (Fig. 8C); tibia I wholly yellow. }\end{array}$ & Haplothrips acanthoscelis \\
\hline \multirow[t]{2}{*}{$24(22)$} & $\begin{array}{l}\text { Maxillary stylets close together, almost } \\
\text { meeting medially (Fig. 8D). }\end{array}$ & Haplothrips utae \\
\hline & $\begin{array}{l}\text { Maxillary stylets at least a third of head's } \\
\text { width apart (Fig. 4E). }\end{array}$ & 25 \\
\hline \multirow[t]{2}{*}{$25(24)$} & $\begin{array}{l}\text { Maxillary stylets half of head's with apart (cf. } \\
\text { Fig. 8C) }\end{array}$ & Haplothrips distinguendus \\
\hline & $\begin{array}{l}\text { Maxillary stylets } 0.3-0.4 \text { of head's with apart } \\
\text { (Fig. } 7 \mathrm{H}) \text {. }\end{array}$ & 26 \\
\hline $26(25)$ & $\begin{array}{l}\text { Antennal segments III-IV, sometimes also V- } \\
\text { VI, brown with yellow base, segments VII- } \\
\text { VIII brown (Fig. 8E). }\end{array}$ & Haplothrips hukkineni \\
\hline
\end{tabular}




\begin{tabular}{|c|c|c|}
\hline & $\begin{array}{l}\text { Antennal segment III shaded yellow to light } \\
\text { brown, IV-VII brown (Fig. 8F). }\end{array}$ & 27 \\
\hline \multirow[t]{2}{*}{27 (26). } & $\begin{array}{l}\text { Anteromarginal setae short, about as long as } \\
\text { discal seta (Fig. 8G). }\end{array}$ & Haplothrips alpester \\
\hline & $\begin{array}{l}\text { Anteromarginal setae long, at least twice as } \\
\text { long as discal seta (cf. Fig. 7G). }\end{array}$ & Haplothrips tritici \\
\hline \multirow[t]{2}{*}{$28(8)$. } & $\begin{array}{l}\text { Fore femur with apical tubercle (Fig. 9A); } 3 \\
\text { sense cones on antennal segment III-IV (Fig. } \\
\text { 9B). }\end{array}$ & Acanthothrips nodicornis \\
\hline & $\begin{array}{l}\text { Fore femur without apical tubercle; if teeth on } \\
\text { femur present then antennal segment IV with } \\
4 \text { sense cones. }\end{array}$ & 29 \\
\hline \multirow[t]{2}{*}{$29(28)$. } & Eyes ventrally elongated (Fig. 9C). & Cephalothrips monilicornis \\
\hline & Eyes not ventrally elongated. & 30 \\
\hline \multirow[t]{2}{*}{30 (29). } & $\begin{array}{l}\text { Mouth cone long and narrow, extending } \\
\text { beyond posterior margin of pronotum (Fig. } \\
\text { 9D). }\end{array}$ & Poecilothrips albopictus \\
\hline & Mouth cone shorter. & 31 \\
\hline \multirow[t]{2}{*}{$31(30)$. } & $\begin{array}{l}\text { Antennal segment III without sense cones } \\
\text { (Fig. 9E). }\end{array}$ & Lispothrips crassipes \\
\hline & $\begin{array}{l}\text { Antennal segment III with at least } 1 \text { sense } \\
\text { cone (Fig. 9F). }\end{array}$ & 32 \\
\hline \multirow[t]{2}{*}{$32(31)$} & Antennal segment III with 1 sense cone. & Liothrips $\mathbf{3 3}$ \\
\hline & $\begin{array}{l}\text { Antennal segment III with } 2-3 \text { sense cones } \\
\text { (Fig. 9F). }\end{array}$ & 34 \\
\hline \multirow[t]{2}{*}{33 (32). } & $\begin{array}{l}\text { Setae } S 1 \text { on tergite IX as long as, or close to } \\
\text { as long as, segment } X \text { (tube) (Fig. } 9 G) \text {. }\end{array}$ & Liothrips austriacus \\
\hline & $\begin{array}{l}\text { Setae S1 on tergite IX closer to or half as long } \\
\text { as segment X (Fig. 10A) }\end{array}$ & Liothrips setinodis \\
\hline \multirow[t]{2}{*}{34 (32). } & $\begin{array}{l}\text { Abdomen clearly bicolored, with at least } \\
\text { segment VIII-IX mostly yellow (Fig. 10B); } \\
\text { segment X (tube) yellow and often with dark } \\
\text { transverse terminal band or shadings, } \\
\text { sometimes also yellow head and pronotum in } \\
\text { micropterous individuals. }\end{array}$ & 35 \\
\hline & $\begin{array}{l}\text { Abdomen uniformly brown or yellow, } \\
\text { sometimes with pale or red markings. }\end{array}$ & 36 \\
\hline \multirow[t]{2}{*}{35 (34). } & Segment VIII-X yellow (Fig. 10B). & Hoplothrips pedicularius \\
\hline & Segment VI-X yellow. & Hoplothrips caespitis \\
\hline \multirow[t]{2}{*}{$36(34)$. } & $\begin{array}{l}\text { Pronotom with } 5 \text { pairs of well-developed } \\
\text { bristles, may be short but stout (Fig. 10C). }\end{array}$ & 37 \\
\hline & $\begin{array}{l}\text { Pronotum with } 4 \text { pairs of well-developed } \\
\text { bristles, anteromarginals not distinctly stouter } \\
\text { than discal seta. }\end{array}$ & 43 \\
\hline 37 (36). & $\begin{array}{l}\text { Postocular setae present but short, shorter or } \\
\text { as long as the width of eye (Fig. 10E); setae } \\
\text { S1 on tergite IX distinctly shorter than half of } \\
\text { the length of segment X (tube) (Fig. 10F). }\end{array}$ & Phlaeothrips $\mathbf{3 8}$ \\
\hline
\end{tabular}




\begin{tabular}{|c|c|c|}
\hline & $\begin{array}{l}\text { Postocular setae well developed and as long } \\
\text { as or longer than the length of eye (Fig. 10F); } \\
\text { setae } S 1 \text { on tergite IX close to or more than } \\
\text { half as long as segment X (Fig. 10G). }\end{array}$ & 41 \\
\hline \multirow[t]{2}{*}{$38(37)$. } & Head with lateral tubercles (Fig. 10H). & 39 \\
\hline & Head without lateral tubercles (Fig. 10D). & 40 \\
\hline \multirow[t]{2}{*}{39 (38). } & $\begin{array}{l}\text { Antennal segment III about } 3 \text { times as long as } \\
\text { wide. Tibia I often wholly yellow (Fig. 10I). }\end{array}$ & Phlaeothrips coriaceus \\
\hline & $\begin{array}{l}\text { Antennal segment III less than } 2.6 \text { times as } \\
\text { long as wide. Tibia I foten only yellow } \\
\text { apically (Fig. 10J). }\end{array}$ & Phlaeothrips denticauda \\
\hline \multirow[t]{2}{*}{$40(38)$. } & $\begin{array}{l}\text { Tibia I largely yellow, tibia II and III } \\
\text { distinctly bicolored with yellow apex and } \\
\text { base (Fig. 10K). }\end{array}$ & Phlaeothrips annulipes \\
\hline & All tibia brown. & Phlaeothrips bispinosus \\
\hline \multirow[t]{2}{*}{41 (37). } & $\begin{array}{l}\text { Postocular setae (Fig. 10E) and setae S1 on } \\
\text { tergite IX expanded apically (Fig. 10G). } \\
\text { Forewing constricted medially. }\end{array}$ & Hoplandrothrips bidens \\
\hline & $\begin{array}{l}\text { Postocular setae and setae S1 on tergite IX } \\
\text { acute. Forewing parallel sided. }\end{array}$ & 42 \\
\hline \multirow[t]{2}{*}{$42(41)$. } & $\begin{array}{l}\text { Large pronotal bristles expanded apically } \\
\text { (Fig. 11A). }\end{array}$ & Holothrips schaubergeri \\
\hline & Large pronotal bristles acute. & Hoplothrips polysticti \\
\hline \multirow[t]{2}{*}{43 (36). } & $\begin{array}{l}\text { Antennal segment IV with } 2 \text { sense cones (Fig. } \\
\text { 9F). }\end{array}$ & 44 \\
\hline & Antennal segment IV with 3-4 sense cones. & 48 \\
\hline \multirow[t]{2}{*}{44 (43). } & $\begin{array}{l}\text { Maxillary stylets about a third of heads width } \\
\text { apart (Fig. 11B). }\end{array}$ & 45 \\
\hline & $\begin{array}{l}\text { Maxillary stylets close together, meeting or } \\
\text { almost meeting medially (Fig. 4B). }\end{array}$ & 46 \\
\hline \multirow[t]{2}{*}{45 (44). } & $\begin{array}{l}\text { Large pronotal bristles expanded apically (cf. } \\
\text { Fig. 11A). }\end{array}$ & Hoplothrips longisetis \\
\hline & Pronotal bristles acute. & Hoplothrips caespitis \\
\hline \multirow[t]{2}{*}{46 (44). } & $\begin{array}{l}\text { Setae S1 as long as or longer than segment } \mathrm{X} \\
\text { (tube) (Fig. 11C). }\end{array}$ & Hoplothrips unicolor \\
\hline & Setae S1 shorter than segment X. & 47 \\
\hline \multirow[t]{2}{*}{47 (46). } & $\begin{array}{l}\text { Antennal segment I slightly tapering apically, } \\
\text { II and III yellow or light brown at least partly, } \\
\text { and segment IV-VII distinctly darker (Fig. } \\
\text { 11D). Macropterous females with cluster of } \\
\text { sensory hairs on antennal segments IV-VI } \\
\text { (Fig. 11D). }\end{array}$ & Hoplothrips semicaecus \\
\hline & $\begin{array}{l}\text { Antennal segment I more evenly tubular, II- } \\
\text { VII more uniformly yellow or light brown } \\
\text { (Fig. 11E). }\end{array}$ & Hoplothrips carpathicus \\
\hline \multirow[t]{2}{*}{48 (44). } & Antennal segment IV with 3 sense cones & 49 \\
\hline & Antennal segment IV with 4 sense cones & 52 \\
\hline
\end{tabular}




\begin{tabular}{|c|c|c|}
\hline \multirow[t]{2}{*}{49 (48). } & $\begin{array}{l}\text { Antennal segment I slightly tapering apically, } \\
\text { II and III yellow or light brown at least partly, } \\
\text { and segment IV-VII distinctly darker (Fig. } \\
\text { 11D). Macropterous females with cluster of } \\
\text { sensory hairs on antennal segments IV-VI } \\
\text { (Fig. 11D). }\end{array}$ & Hoplothrips semicaecus \\
\hline & Antennal segments different. & 50 \\
\hline \multirow[t]{2}{*}{50 (49). } & $\begin{array}{l}\text { Setae S1 on tergite IX blunt apically (Fig. } \\
\text { 11F). }\end{array}$ & Thorybothrips unicolor \\
\hline & Setae S1 on tergite IX acute (Fig. 11G). & 51 \\
\hline \multirow[t]{2}{*}{$51(50)$. } & $\begin{array}{l}\text { Setae S1 on tergite IX as long as or longer } \\
\text { than tube (Fig. 11C). }\end{array}$ & Hoplothrips unicolor \\
\hline & $\begin{array}{l}\text { Setae S1 on tergite IX shorter than tube (Fig. } \\
11 \mathrm{G}) \text {. }\end{array}$ & Hoplothrips polysticti \\
\hline \multirow[t]{2}{*}{$52(48)$. } & $\begin{array}{l}\text { Major pronotal bristles expanded (cf. Fig } \\
11 \mathrm{~A} \text { ). }\end{array}$ & $\begin{array}{r}\text { Hoplandrothrips } \\
\text { williamsianus }\end{array}$ \\
\hline & $\begin{array}{l}\text { Major pronotal bristles acute or blunt, not } \\
\text { expanded. }\end{array}$ & 53 \\
\hline \multirow[t]{2}{*}{$53(52)$} & $\begin{array}{l}\text { Antennal segment III with long and strongly } \\
\text { inwards curving sense cone at inner margin } \\
\text { (Fig. } 11 \mathrm{H} \text { ). }\end{array}$ & Hoplothrips fungi \\
\hline & $\begin{array}{l}\text { Sense cones on segment III forward pointing } \\
\text { and stout. }\end{array}$ & 54 \\
\hline \multirow[t]{2}{*}{$54(53)$. } & $\begin{array}{l}\text { Antennal segments IV-VI brown, IV at most } \\
\text { slightly shaded (Fig. 11D). }\end{array}$ & 55 \\
\hline & $\begin{array}{l}\text { Antennal segments IV-VI bicolored with } \\
\text { basal half yellow (Fig. 11I). }\end{array}$ & 56 \\
\hline \multirow[t]{2}{*}{$55(54)$} & $\begin{array}{l}\text { Antennal segment VIII not distinctly } \\
\text { constricted at base, VII and VIII confluent } \\
\text { (Fig. 11D). }\end{array}$ & Hoplothrips semicaecus \\
\hline & $\begin{array}{l}\text { Antennal segment VIII constricted at base, } \\
\text { separating VII and VIII (Fig. 11J). }\end{array}$ & Hoplothrips polysticti \\
\hline \multirow[t]{2}{*}{$56(54)}$. & All tibia wholly yellow (Fig. 11K). & Hoplothrips corticis \\
\hline & $\begin{array}{l}\text { Only tibia I yellow, tibia II and III at most } \\
\text { yellow basally and apically (Fig. 11L). }\end{array}$ & Hoplothrips ulmi \\
\hline
\end{tabular}

\section{Checklist of the Swedish Phlaeothripidae}

\section{Idolothripinae Bagnall, 1908}

\section{Diagnosis}

The Idolothripinae are distinguished by the broad maxillary stylets. The maxillary stylets are at least $5 \mu \mathrm{m}$ broad.

\section{Notes}

There are 7 known species in Sweden in this subfamily. The broad maxillary stylets are hypothesized to be an adaptation to feeding on fungal spores (Mound and Palmer 1983). 
Bacillothrips Buffa, 1908

Bacillothrips nobilis (Bagnall, 1909)

Distribution: Go.

Remark: First record for Sweden. In Fennoscandia this species has previously been recorded from Denmark, Norway and Finland (Kobro 2011, Gertsson 2015b). Feeding on fungal spores (Mound 1974), and found in dry grass, sedges, and dead branches mainly from Salix L. (Schliephake and Klimt 1979; Mound et al. 1976).

Material examined: SWEDEN • 1 q Gotland, Gotlands kommun, Vitärtskällan; $57.8512^{\circ} \mathrm{N}$, $18.8123^{\circ} \mathrm{E}$; $10 \mathrm{Jul} .2011$; B. Eklund, leg.; Malaise trap; Loc. 029-06.

Bolothrips Priesner, 1926

Bolothrips bicolor Heeger, 1852

Distribution: Up.

Bolothrips dentipes (O. M. Reuter, 1880)

Distribution: Sk, Sm, Öl, Ög, Bo, Sö, Up, Lu.

Bolothrips icarus (Uzel, 1895)

Distribution: Sk, Öl, Go, GS, Sö, Up.

Remark: First record for Sö.

Material examined: SWEDEN • 1q Södermanland, Nyköping kommun, Skeppsvik; dry meadow at roadside with Crepis, Vicia and Plantago; $58.639919^{\circ} \mathrm{N}, 16.822483^{\circ} \mathrm{E} ; 3 \mathrm{Jun}$ 2021; E. Wahlberg, leg.

Cryptothrips Uzel, 1895

Cryptothrips nigripes (O. M. Reuter, 1880)

Distribution: Sk, Sm, Öl, Bo, Sö, Up, Vs, Vr, Dr, Lu.

Megathrips Targioni-Tozzetti, 1881

Megathrips lativentris (Heeger, 1852)

Distribution: Sk, B1, Sm, Öl, Go, GS, Ög, Vg, Bo, Ds, Nä, Sö, Up, Vr, Dr, Gä, Hs, Me, Hr, Jä, Ån, Vb, Nb, Ly, Pi, Lu, To.

Megalothrips Uzel, 1895

Megalothrips bonanni Uzel, 1895 
Distribution: Sk.

\section{Phlaeothripinae Uzel, 1895}

Diagnosis

Differentiated from Idolothripinae by the slender maxillary stylets, at most $3 \mu \mathrm{m}$ wide.

Notes

The majority of the species in the family belongs to this subfamily, for Sweden 42 species are known. The life histories are very varying, ranging from species feeding on fungal hyphae to predatory species (Mound and Tree 2020).

Acanthothrips Uzel, 1895

Acanthothrips nodicornis (O. M. Reuter, 1880)

Distribution: Sm, Bo, Sö, Up, Vs, Vr, Dr.

Cephalothrips Uzel, 1895

Cephalothrips monilicornis (O. M. Reuter, 1880)

Distribution: Sk, Vg, Öl, Sm, Sö, Up, Vr.

Remark: First record for Vg.

Material examined: SWEDEN • $2 q q$ Västergötland, Laxå kommun, Finnerödja; sandy slope with Carex and Calluna; 58.929699 ${ }^{\circ}$ N, $14.340039^{\circ}$ E; 5 Jun 2021; E. Wahlberg, leg.

Haplothrips Amyot \& Serville, 1843

Haplothrips acanthoscelis (Karny, 1910)

Distribution: Sk, Öl.

Haplothrips aculeatus (Fabricius, 1803)

Distribution: Sk, B1, Ha, Sm, Öl, Go, Ög, Vg, Bo, Sö, Up.

Remarks: First record for Ha.

Material examined: SWEDEN • 1 q Halland, Varberg kommun, Tvååker; meadow on old cultivated land with Quercus, Fagus and Fraxinus; $57.02078^{\circ}$ N, $12.47949^{\circ}$ E; 19 May 2021;

E. Wahlberg, leg.

Haplothrips alpester Priesner, 1914

Distribution: Sk, Öl, Vg, Sö, Vr, Ly. 
Remark: First record for Sö.

Material examined: SWEDEN • $1 \circlearrowright^{\Uparrow}$ Södermanland, Nyköping kommun, Skeppsvik; marsh with Hierochloë, Juncus, Carex, Luzula and Schoenoplectus; $58.645574^{\circ} \mathrm{N}, 16.843128^{\circ} \mathrm{E} ; 3$ Jun 2021; E. Wahlberg, leg.

Haplothrips alpicola Priesner, 1950

Distribution: Ly.

Haplothrips angusticornis Priesner, 1921

Distribution: Sk, Ög, Up, Vr.

Haplothrips distinguendus (Uzel, 1895)

Distribution: Sk, Sm, Vg, Up.

Haplothrips hukkineni Priesner, 1939

Distribution: Sk, Sm, Öl, Go Sö, Up, Vr.

Haplothrips leucanthemi (Schrank, 1781)

Distribution: Sk, Ha, Sm, Öl, Bo, Ds, Nä, Ög, Sö, Up, Vr, Jä, Vb, Lu, To.

Remark: First record for Ha.

Material examined: SWEDEN • $1{ }^{\Uparrow}$ Halland, Halmstad kommun, Särdal; on Armeria maritima; $56.73674^{\circ} \mathrm{N}, 12.64723^{\circ} \mathrm{E}$; 19 May 2021; E. Wahlberg, leg.

Haplothrips minutus (Uzel, 1895)

Distribution: Sk, Sö.

Haplothrips propinquus Bagnall, 1933

Distribution: Sk, Sö, Up, Vr, Gä, Hs, Jä, Lu.

Haplothrips senecionis Bagnall, 1932

Distribution: Öl.

Haplothrips setiger Priesner, 1921

Distribution: Sk.

Haplothrips statices (Haliday, 1836)

Distribution: Sk, Bl, Ha, Sm, Öl, Ög, Bo, Sö, Up, Hs, Hr, Jä, Ån, Nb, Lu. 
Haplothrips subtilissimus (Haliday, 1852)

Distribution: Sk, Sm, Sö, Up.

Remark: First record for Sm.

Material examined: SWEDEN • 1 \& Småland, Kalmar kommun, Bottorp; alley with Quercus and Prunus; 56.591923 ${ }^{\circ}$, $16.212710^{\circ} \mathrm{E} ; 11$ May 2021; E. Wahlberg, leg.

Haplothrips tritici (Kurdjumov, 1912)

Distribution: Sö.

Haplothrips utae Klimt, 1970

Distribution: Sk, Sm.

Haplothrips verbasci Osborn, 1896

Distribution: Sk.

Holothrips Karny, 1911

Holothrips schaubergeri (Priesner, 1920)

Distribution: Sö.

Hoplandrothrips Hood, 1912

Hoplandrothrips bidens (Bagnall, 1910)

Distribution: Sk, Öl, Up.

Hoplandrothrips williamsianus Priesner, 1923

Distribution: Vr.

Hoplothrips Amyot \& Serville, 1843

Hoplothrips caespitis (Uzel, 1895)

Distribution: Sk.

Hoplothrips carpathicus Pelikan, 1961

Distribution: Sk, Ds, Sö, Up, Vr.

Hoplothrips corticis (De Geer, 1773) 
Distribution: Sk, Bl, Öl, Go, GS, Sm, Ög, Bo, Sö, Up, Vb, Nb.

Remark: First record for Nb.

Material examined: SWEDEN • 19 Norrbotten, Åsele, Björnlandet nationalpark; $63.9702^{\circ} \mathrm{N}$, $18.0533^{\circ} \mathrm{E} ; 12-26$ Jul 2011; K. Norberg, B.O. Johansson, leg.; Malaise trap; Loc. 034-04.

Hoplothrips fungi (Zetterstedt, 1828)

Distribution: B1, Öl, Go, Up, Hs.

Hoplothrips longisetis (Bagnall, 1910)

Distribution: Sk, Ds, Vr.

Hoplothrips pedicularius (Haliday, 1836)

Distribution: Sk, Sm, Sö, Up, Vr, Dr, Hs.

Hoplothrips polysticti (Morison, 1949)

Distribution: Sk, Ög, Vr, Dr, Vb.

Hoplothrips semicaecus (Uzel, 1895)

Distribution: Sk, Ha, Up.

Remark: First record for Ha.

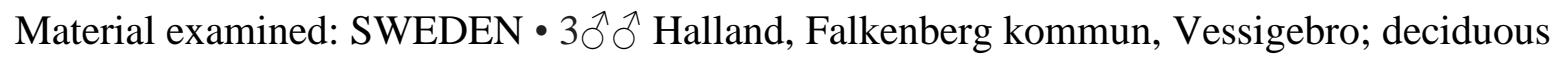
forest (Fagus), in Fomes fomentarius; $57.05748^{\circ} \mathrm{N}, 12.78881^{\circ} \mathrm{E}$; 18 May 2021; E. Wahlberg, leg.

Hoplothrips ulmi (Fabricius, 1781)

Distribution: Sk, Bl, Ha, Sm, Öl, Go, GS, Ög, Bo, Ds, Sö, Up, Vs, Vr, Dr, Ån, Vb, Lu.

Hoplothrips unicolor (Vuillet, 1914)

Distribution: Sö.

Liothrips Uzel, 1895

Liothrips austriacus (Karny, 1910)

Distribution: Vr.

Liothrips setinodis (O. M. Reuter, 1880)

Distribution: Ha, Up. 
Lispothrips O. M. Reuter, 1899

Lispothrips crassipes (Jablonowski, 1894)

Distribution: Sm.

Phlaeothrips annulipes O. M. Reuter, 1880

Distribution: Sk, Sm, Ög, Bo, Sö, Up, Vs, Vr, Dr, Vb.

Phlaeothrips bispinosus Priesner, 1919

Distribution: Vr.

Phlaeothrips coriaceus Haliday, 1836

Distribution: Sk, Bl, Ha, Sm, Öl, Go, Bo, Sö, Up, Vs, Hs, Vb.

Phlaeothrips denticauda Priesner, 1914

Distribution: Sk, Vr.

Poecilothrips Uzel, 1895

Poecilothrips albopictus Uzel, 1895

Distribution: Sk, Vr.

Thorybothrips Priesner, 1924

Thorybothrips unicolor (Schille, 1911)

Distribution: Ö1, Go.

Xylaplothrips fuliginosus (Schille, 1911)

Distribution: Sm, Ha, Sö, Ds, Vr, Dr, Lu, To.

Remark: First record for Ha and Sö.

Material examined: SWEDEN • $1{ }^{\Uparrow}$ Halland, Falkenberg kommun, Vessigebro; on Larix decidua, $56.97484^{\circ} \mathrm{N}, 12.72877^{\circ} \mathrm{E}, 19$ May 2021; E. Wahlberg, leg. $\bullet 2 \circ$ 9 Södermanland, Nyköping kommun, Skeppsvik; mixed forest; $58.645574^{\circ} \mathrm{N}, 16.843128^{\circ} \mathrm{E}$; 03 Jun 2021; E. Wahlberg, leg. $\bullet 19$ Södermanland, Gnesta kommun, Fridsta; private garden with mixed vegetation; $59.067287^{\circ} \mathrm{N}, 17.155016^{\circ} \mathrm{E}$; $14-21$ Jun 2021 ; E. Wahlberg, leg., window trap. • $1{ }^{\top}$ Södermanland, Gnesta kommun, Önnersta; on dead Betula; $59.047010^{\circ} \mathrm{N}, 17.145951^{\circ} \mathrm{E}$; 16 Jul 2021; E. Wahlberg, leg. 


\section{Acknowledgments}

We thank Andrea Hastenpflug-Vesmanis (Forschungsinstitut und Naturmuseum Senckenberg, Germany), Christoffer Fägerström (Lund Museum of Zoology, Sweden), Manfred R. Ulitzka (Offenburg, Germany) and Sverre Kobro (Norwegian Institute of Bioeconomy Research, Norway) for their help in providing material used in this study. This study is part of a project funded by the Swedish Taxonomy Initiative (SLU.dha.2020.4.3-228).

\section{References}

Ahlberg O (1926) Tripsar. Thysanoptera. Svensk insektsfauna 6. Sveriges entomologiska förening, Stockholm. 62 pp.

Amyot CJB, Audinet-Serville JG (1843) Histoire Naturelle des Insectes. Hémiptères. Librairie encyclopédique de Roret, Paris, 657pp.

Bagnall RS (1908) On some new and curious Thysanoptera (Tubulifera) from Papua. Annals and Magazine of Natural History Series 8 1: 355-363.

Bagnall RS (1909) Notes on some Thysanoptera (Tubulifera) new to the British fauna, with description of a new species of Megathrips. Entomologist's monthly Magazine 45: 129-132.

Bagnall RS (1910) On a small collection of Thysanoptera from Hungary. Annales HistoricoNaturales Musei Nationalis Hungarici 8: 372-376.

Bagnall RS (1932) On five new species of Thysanoptera from Great Britain. Entomologist's monthly Magazine 68: 161-165.

Bagnall RS (1933) Contributions towards a knowledge of the European Thysanoptera IV. Annals and Magazine of Natural History 11: 647-661.

Boyd Jr. BW, Held DW (2006) Androthrips ramachandrai (Thysanoptera: Phlaeothripidae): an introduced thrips in the United States. Florida Entomologist 89: 455-458.

Buffa P (1908) Esame della raccolta di Tisanotteri italiani esistenti nel Museo Civico di Storia Naturale di Genova. Redia 4: 382-91.

De Geer, C 1773. Mémoires pour servir à l'histoire des insectes. Tome troisième. Hesselberg, Stockholm, $750 \mathrm{pp}$.

Fabricius JC (1781) Species insectorvm exhibentes eorvm differentias specificas, synonyma avctorvm, loca natalia, metamorphosin adiectis observationibvs, descriptionibvs. C. E. Bohnii, Hamburg and Kiel, 517 pp.

Fabricius JC (1803) Systema Rhyngotorum secundum ordines, genera, species adiectis synonymis, locis, observationibus, descriptionibus. Carolus Reichard, Braunschweig, 314 pp.

Gertsson C-A (2015a) Nya intressanta tripsarter från Öland. Lucanus 20: 36-39.

Gertsson C-A (2015b) An annotated checklist of Thysanoptera (thrips) from the Nordic countries. Entomologisk Tidskrift 136: 185-198. 
Gertsson C-A (2021) Två för Sverige nya tripsarter: Haplothrips alpicola Priesner, 1950 och Haplothrips utae Klimt, 1970 (Thysanoptera) samt nya provinsfynd. Entomologisk Tidskrift 142: $21-30$.

Gertsson C-A, Fägerström C (2017) Två nya tripsarter (Thysanoptera) för Sverige samt tre nya landskapsfynd från Öland. Entomologisk Tidskrift 138: 131-136.

Gertsson C-A, Fägerström C, Sjödahl M (2022) Två för Skandinavien nya tripsarter (Thysanoptera): Hoplothrips caespitis (Uzel, 1895) och Megalothrips bonannii Uzel, 1895 samt nya provinsfynd. Entomologisk Tidskrift 143, in print.

Haliday AH (1836) An epitome of the British genera in the Order Thysanoptera with indications of a few of the species. Entomological Magazine 3: 439-451.

Haliday AH (1852) Order III Physapoda. In: Walker F. List of the Homopterous insects in the British Museum Part IV. British Museum, London, 1094-1118.

Heeger E (1852) Beitrage zur Insecten-Fauna Osterrreichs. Sitzungsberichte der mathematisch-naturwissenschaftlichen Classe kaiserlichen Akademie der Wissenschaften, Wien 9: 473-490.

Held DW, Boyd D, Lockley T, Edwards GB (2005) Gynaikothrips uzeli (Thysanoptera: Phlaeothripidae) in the southeastern United States: distribution and review of biology. The Florida Entomologist 88: 538-540.

Hood JD (1912) Descriptions of new North American Thysanoptera. Proceedings of the Entomological Society of Washington 14: 129-160.

Jablonowski J (1894) Thysanoptera nova. Termeszettudomanyi Fuzetek 17: 44-47.

Karny H (1910) Neue Thysanopteren der Wiener Gegend. Mitteilungen des

Naturwissenschaftlichen Vereins an der Universität Wien 8: 41-57.

Karny H (1911) Neue Phloeothripiden-Genera. Zoologischer Anzieger 38: 501-504.

Kirk WDJ (1996) Thrips. Naturalists' Handbooks 25. The Richmond Publishing C. Ltd., Slough, $70 \mathrm{pp}$.

Klimt K (1970) Über eine neue, feuchte Standorte bewohnende Haplothrips-Art (Thysanoptera) - Haplothrips utae spec. nov. Entomologische Nachrichten 13: 121-128.

Kobro S (2011) Checklist of Nordic Thysanoptera. Norwegian Journal of Entomology 58: 2026.

Kobro S (2013) Norske Insekttabeller 19. Trips (Thysanoptera). Norsk Entomologisk Forening, Oslo, $49 \mathrm{pp}$. 
Kobro S, Rafoss T (2006) Identification of adult males and females of Hoplothrips species (Thysanoptera: Tubulifera) known from Norway, and some deductions on their life history. Entomologica Fennica 17: 184-192.

Kucharczyk H, Zawirska I (1994) Study on the thrips fauna (Insecta: Thysanoptera) on xerothermic grassland of South-East Poland. Sonderdruck aus CFS-Courier 178: 3-7.

Kucharczyk H (2004) Thrips (Insecta: Thysanoptera) as an element of ecological monitoring in Białowieża Primeval Forest. Leśne Prace Badawcze 3: 85-94.

Kucharczyk H, Kucharczyk M (2008) The red list of threatened thrips species of middleeastern Poland. Acta Phytopathologica et Entomologica Hungarica 43: 297-305.

Kurdjumov (1912) Two Anthothrips injurious to cereals. With description of a new species. Poltava Trd selisk-choz opytn stancii 6: 1-44.

Morison (1949) Thysanoptera of the London Area. Part III. Supplement to London Naturalist 28: $76-131$.

Moritz G (2006) Thripse - Fransenflügler, Thysanoptera. Pflanzensaftsaugende Insekten Bd. 1. Westarp Wissenschaften, Hohenwarsleben, 384 pp.

Mound LA (1974) The Nesothrips complex of spore-feeding Thysanoptera (Phlaeothripidae: Idolothripinae). Bulletin of the British Museum (Natural History) Entomology 31: 109-188.

Mound LA (2004) Thysanoptera - Diversity and Interactions. Annual Review of Entomology 50: $247-269$.

Mound LA, Morison GD, Pitikin BR, Palmer JM (1976) Thysanoptera. Handbooks for the identification of British Insects, Vol. I. Part 1. Royal Entomological Society, London, 79 pp.

Mound LA, Palmer JM (1983) The generic and tribal classification of spore-feeding Thysanoptera (Phlaeothripidae: Idolothripinae). Bulletin of the British Museum (Natural History) Entomology 46: 1-174.

Mound LA, Tree DJ (2021) Thysanoptera Australiensis - Thrips of Australia. Lucidcentral.org, Identic Pty Ltd, Queensland, Australia.

Osborn H (1896) Notes on a new species of Phleothrips, with description. Proceedings of the Iowa Academy of Sciences 3: 228.

Paine TD (1992) Cuban laurel thrips (Thysanoptera: Phlaeothripidae) biology in Southern California: seasonal abundance, temperature dependent development, leaf suitability, and predation. Annals of the Entomological Society of America 85: 164-172.

Pelikan (1961) New species of Thysanoptera from Czechoslovakia - IV. Časopis Československé Společnosti Entomologické (Acta Societatis Entomologicae Cechosloveniae) 58: $60-70$. 
Preisner H (1914) Neue Thysanopteren (Blasenfüße) aus Oesterreich. Entomologische Zeitschrift 27: 259-261.

Priesner H (1919) Zur Thysanopteren-Fauna Albaniens. Sitzungsberichte der Kaiserlichen Akademie der Wissenschaften 128: 115-144.

Priesner H (1920) Kurze Beschreibung neuer Thysanopteren aus Österreich. Sitzungsberichte der Kaiserlichen Akademie der Wissenschaften 129: 71-88.

Priesner H (1921) Haplothrips-Studien. Treubia 2: 1-20.

Priesner H (1923) Ein Beitrag zur Kenntnis der Thysanopteren Surinams. Tijdschrift voor Entomologie 66: 88-111.

Priesner H (1924) Neue Thysanopteren. Sitzungsberichte der Kaiserlichen Akademie der Wissenschaften 133: 527-542.

Priesner, H (1926) Die Jugendstadien der Malayischen Thysanopteren. Treubia 8: 1-264.

Priesner H (1939) Thysanopterologica VII. Konowia 17: 266-270.

Priesner H (1950) Further studies in Haplothrips and allied genera (Thysanoptera). Bulletin de la Societe Royale entomologique d'Egypte 34: 69-120.

Quick U (1977) New records and notes on the Swedish Thrips fauna (Thysanoptera). Entomologisk Tidskrift 98: 127-131.

Reuter OM (1880) Thysanoptera Fennica, I, Tubulifera. Bidrag till Kannedom af Finlands Natur och Folk 40: 1-26.

Reuter OM (1899) Thysanoptera Fennica. Acta Societas pro Fauna et Flora Fennica 17: 1-67.

Schille F (1911) Thysanopterorum genera et species novae. Sprawozdanie Komisyi Fizyograficznej 45: 1-10.

Schliephake G, Klimt K (1979). Thysanoptera, Fransenflügler. Die Tierwelt Deutschlands. Teil 66. Gustav Fisher Verlag, Jena, 477 pp.

Schrank FvP (1781) Enumeratio Insectorum Austriae indigenorum. Klett, Augsburg, 548 pp.

Sörensson M (2012) Pilotinventering av den saproxyliska insektsfaunan i Dalby Söderskog 2008. Länsstyrelsen i Skåne, Malmö, 75 pp.

Targioni-Tozzetti A (1881) Relazione intoro ai lavori della R. Stazionedi entomologia agraria di Firenze per gli anni 1877-78. Art. V. Fisapodi (Thrips). Annali di Agricoltura 34: 120-134.

Uzel J (1895) Monographie der Ordnung Thysanoptera. B. E. Tolman, Hradec Králové, 530 pp. 
Vasiliu-Oromulu L, zur Strassen R. Larsson H (2000) New thrips species (Cl. Insecta: Ord. Thysanoptera) for the fauna of Sweden. Review Roumain de Biologie Animals 45: 125-135.

Vuillet A (1914) Description d'un Trichothrips nouveau d'Algerie. Bulletin de la Societe Entomologique Paris 83: 313-315.

Wahlberg E, Johanson KA (2018) Molecular phylogenetics reveals novel relationships within Empidoidea (Diptera). Systematic Entomology 43: 619-636.

Wahlberg E (2019) Revision and morphological analysis of the Ragadidae (Insecta, Diptera). European Journal of Taxonomy 521.

Zetterstedt JW (1828) Fauna insectorum Lapponica, Voss, Leipzig, 557 pp.

\section{Figure legends}

Figure 1. Haplothrips leucanthemi in flower of Leucanthemum vulgare Lam.

Figure 2. Map of Swedish faunistic provinces.

Figure 3. Habitus of Haplothrips utae, dorsal view. Roman numbers indicate abdominal segment number. Scale bar: $100 \mu \mathrm{m}$.

Figure 4. A-D. Head, dorsal view. A. Bolothrips icarus. B. Hoplothrips carpathicus. C. Bacillothrips nobilis (ocellar triangle). D. Cryptothrips nigripes. Scale bars: $100 \mu \mathrm{m}$, bps: base of postocular seta, ms: maxillary stylet.

Figure 5. A-B. Head, dorsal view. A. Bolothrips dentipes (ventral margins of eyes also visible). B. Haplothrips senecionis. C. Habitus, dorsal view, Bacillothrips nobilis. D-E. Pelta D. B. nobilis. E. Megalothrips bonanni. F. Foreleg, Megathrips lativentris. Scale bars: 100 $\mu \mathrm{m}(\mathrm{A}-\mathrm{B}, \mathrm{D}-\mathrm{E}), 1 \mathrm{~mm}(\mathrm{C})$, mb: maxillary bridge, ps: postocular seta, lwp: lateral wing of pelta.

Figure 6. A-C. Part of antenna, dorsal view, roman numbers indicate segment number. A. Xylaplothrips fuliginosus (Schille, 1911). B. Haplothrips subtilissimus (Haliday, 1852). C. Segment IV, ventral view, $H$. subtilissimus. D-F. Dorsal view of right side of head. D. Haplothrips subtilissimus. E. H. alpester. F. H. leucanthemi. Scale bars: $100 \mu \mathrm{m}$, sc: sense cone, br: bristle, ps: postocular seta.

Figure 7. A-B. Dorsal view of segment X (tube). A. H. alpester. B. H. aculeatus. C. Head, dorsal view, H. statices. D. Tergite IX, dorsal view, Haplothrips leucanthemi. E-F. Antenna, dorsal view, antennal segment IV marked. E. H. leucanthemi. F. H. propinquus. G. Pronotum, dorsal view, $H$. alpicola. H. Head dorsal view, $H$. tritici. Scale bars: $100 \mu \mathrm{m}$, ms: maxillary stylet, S1: seta 1, am: anteromarginal seta, ds: discal seta, ps: postocular seta.

Figure 8. A. Distal portion of forewing, $H$ setiger. B-D. Dorsal view of head. B. Haplothrips verbasci. C. H. acanthoscelis $\mathbf{D}$. H. utae. E-F. Antenna, roman numbers indicate segment number. E. H. hukkineni. F. H. tritici. G. Pronotum, dorsal view, H. alpester. Scale bars: 100 $\mu \mathrm{m}$, ps: postocular seta, ms: maxillary stylet, am: anteromarginal seta, ds: discal seta. 
Figure 9. A. Foreleg, Acanthothrips nodicornis. B. Antennal segment IV, A. nodicornis. C. Head, ventral view, Cephalothrips monilicornis. D. Head and pronotum with mouth cone (mesonotum detached), Poecilothrips albopictus. E-F. Antenna, roman numbers indicate segment number. E. Lispothrips crassipes. F. Hoplothrips longisetis. G. Abdominal segments IX-X, yellow structure underneath is male genitalia, Liothrips austriacus. Scale bars: $100 \mu \mathrm{m}$, sc: sense cone, mc: mouth cone, S1: seta 1.

Figure 10. A. Abdominal segments IX-X, Liothrips setinodis. B. Abdomen, dorsal view, roman numbers indicate segment number, Hoplothrips pedicularius. C. Pronotom, dorsal view, Phlaeothrips annulipes. D-E. Left half of head, dorsal view. D. P. annulipes. E. Hoplandothrips bidens. F-G. Abdominal segments IX-X. F. P. annulipes. G. H. bidens. H. Head, dorsal view, Phlaeothrips coriaceus. I-K. Foreleg, dorsal view. I. P. coriaceus. J. P. denticauda. K. Middle leg, P. annulipes. Scale bars: $100 \mu \mathrm{m}, \mathrm{S} 1$ : seta 1, am: anteromarginal seta, ds: discal seta, ps: postocular seta.

Figure 11. A. Anterolateral portion of pronotum, dorsal view, Holothrips schaubergeri. B. Head, dorsal view, Hoplothrips longisetis. C. Segments IX-X, H. unicolor. D-E. Antenna, roman numbers indicate segment number. D. H. semicaecus. E. H. carpathicus. F-G. Segments IX-X. F. Thorybothrips unicolor. G. Hoplothrips polysticti. H. Antenna, segment III, $H$. fungi. I. Antenna, $H$. ulmi. J. Antenna, segments V-VIII, $H$. polysticti. K-L. Middle leg. K. H. corticis. L. H. ulmi. Scale bars: $100 \mu \mathrm{m}$, aa: anteroangular seta, ms: maxillary stylet, sc: sense cone. 


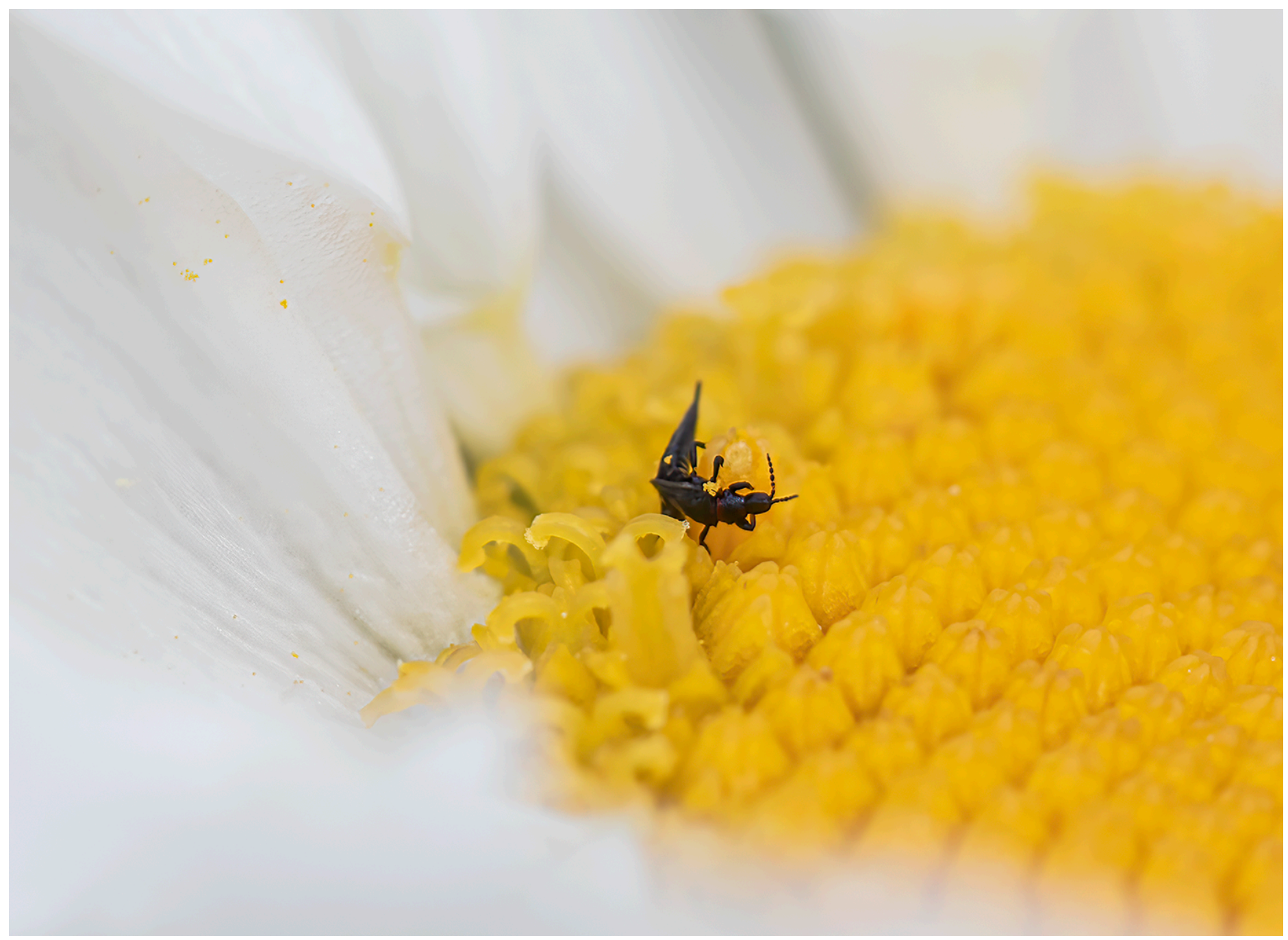




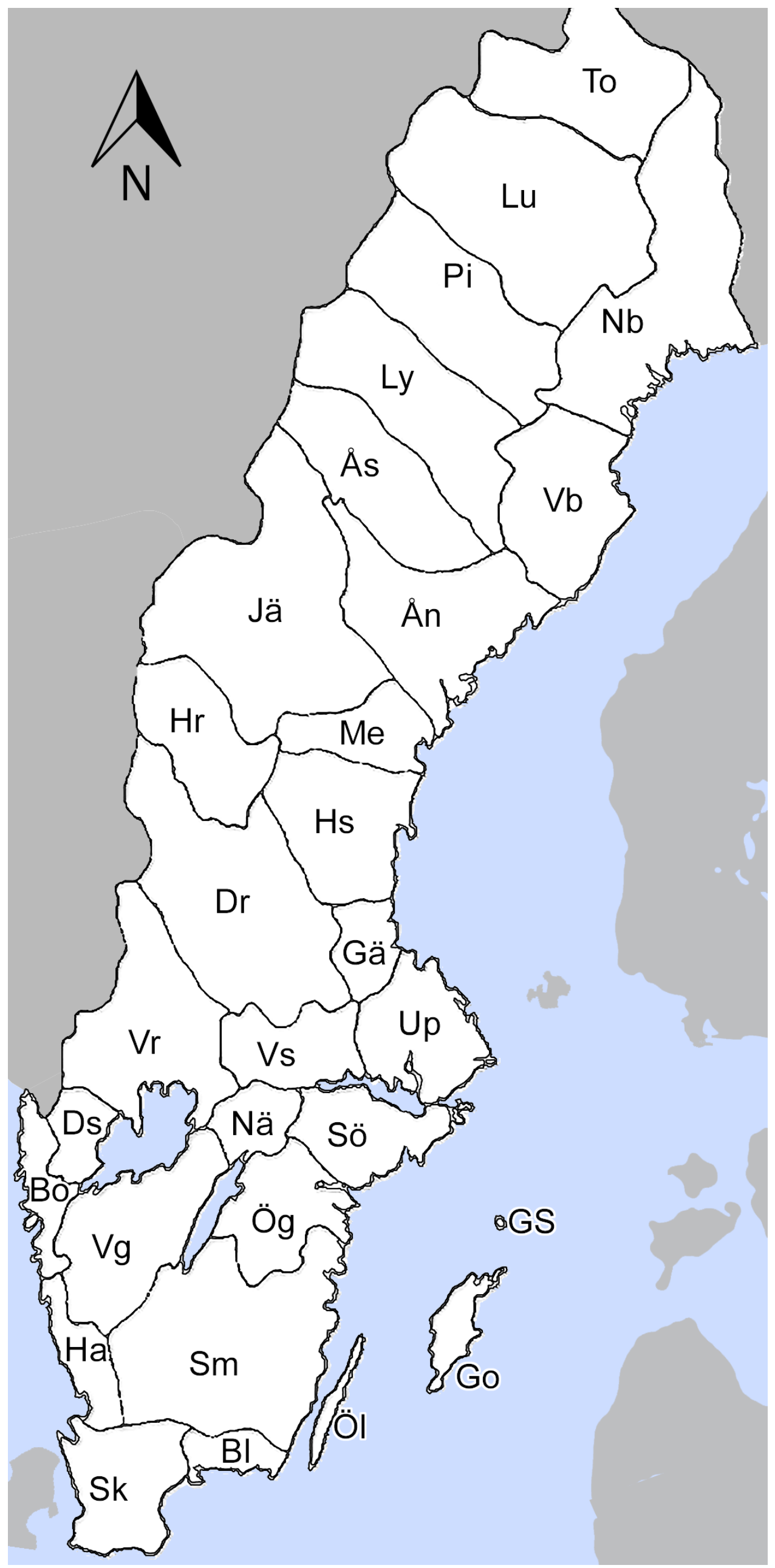



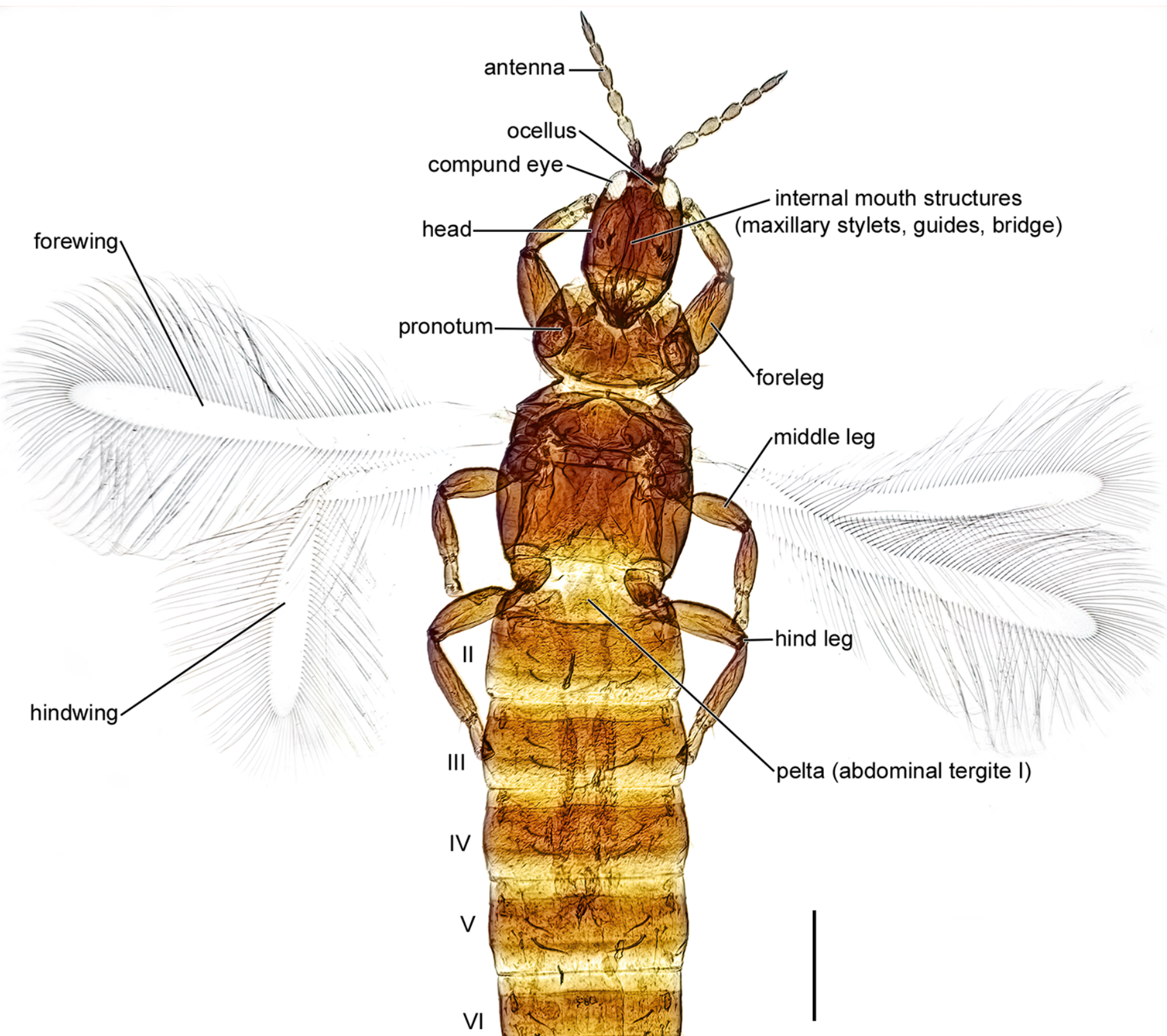

VII

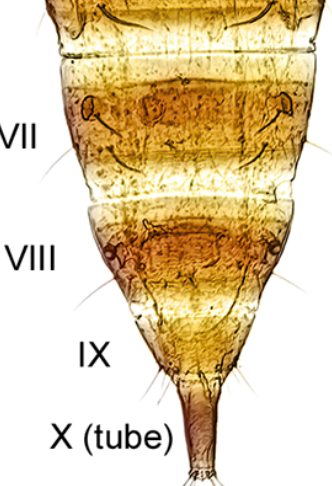



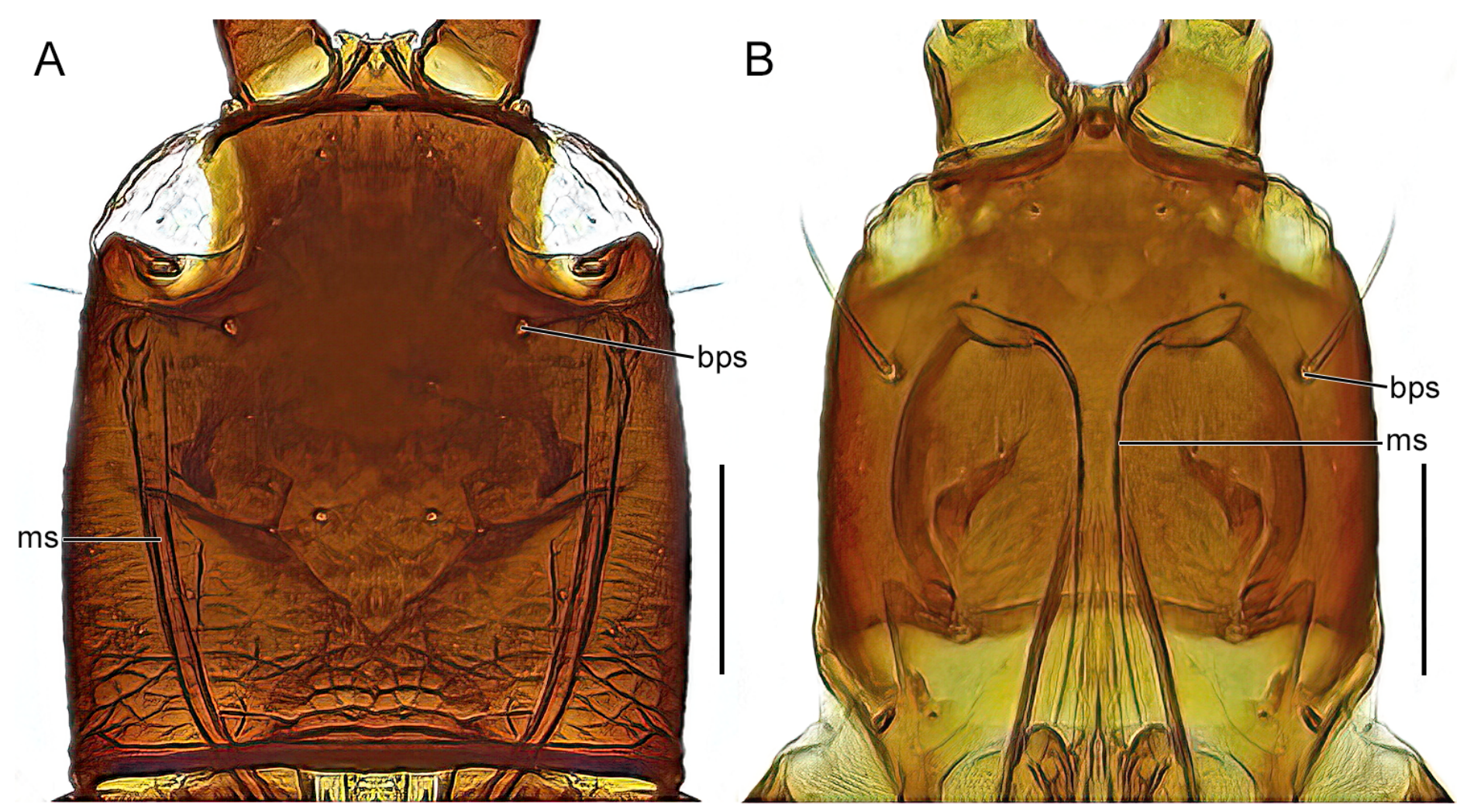

C
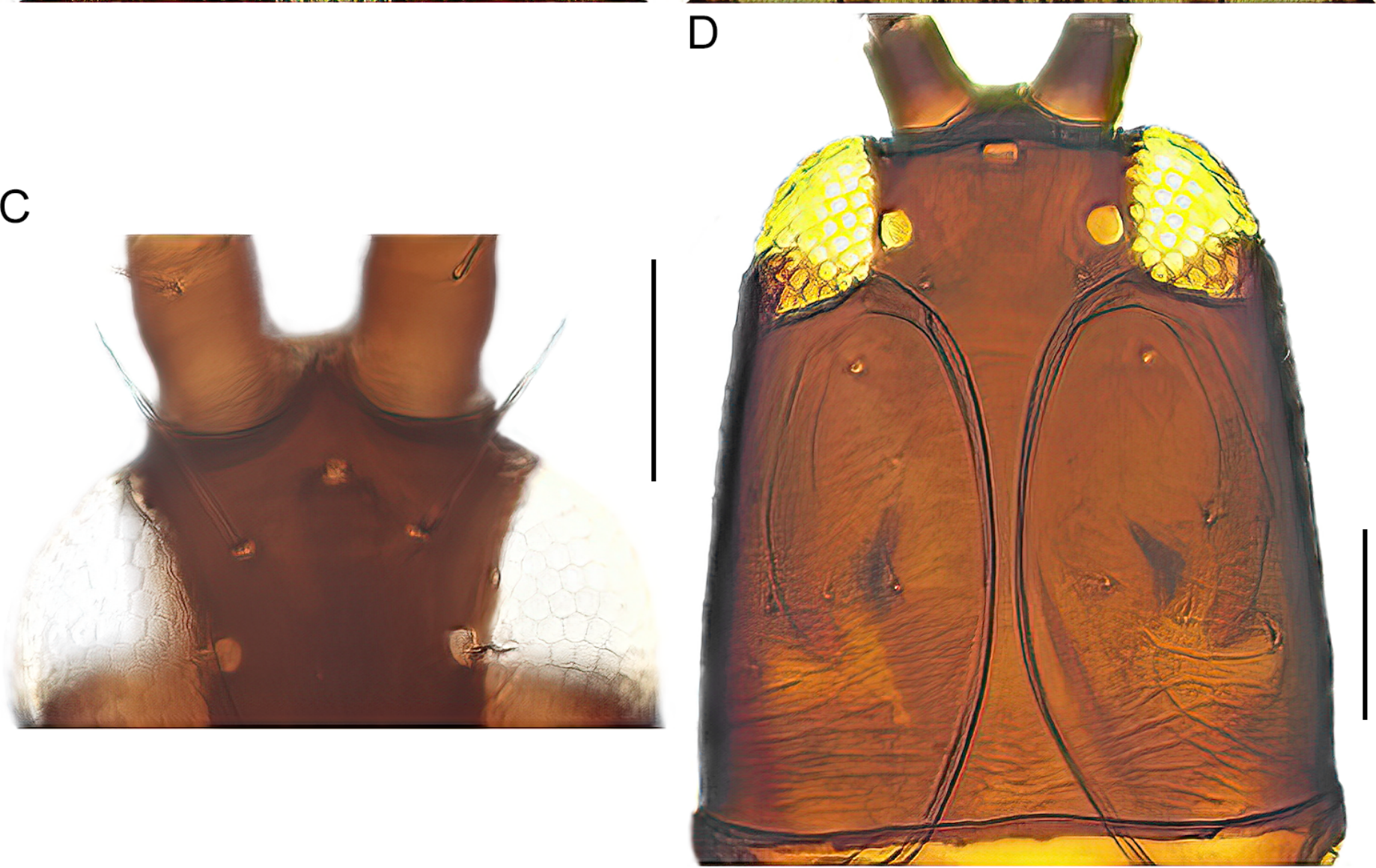

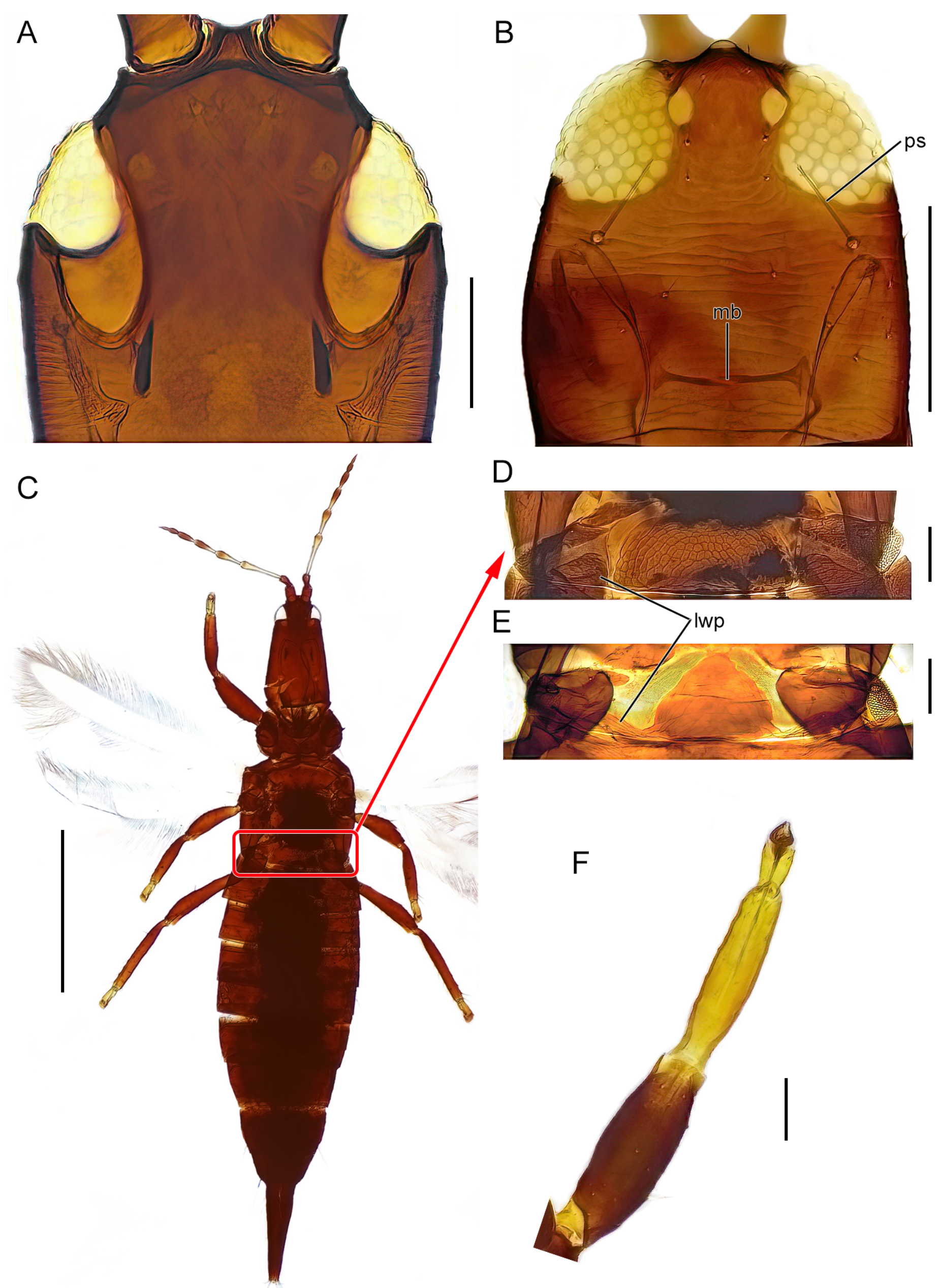

$\mathrm{D}$
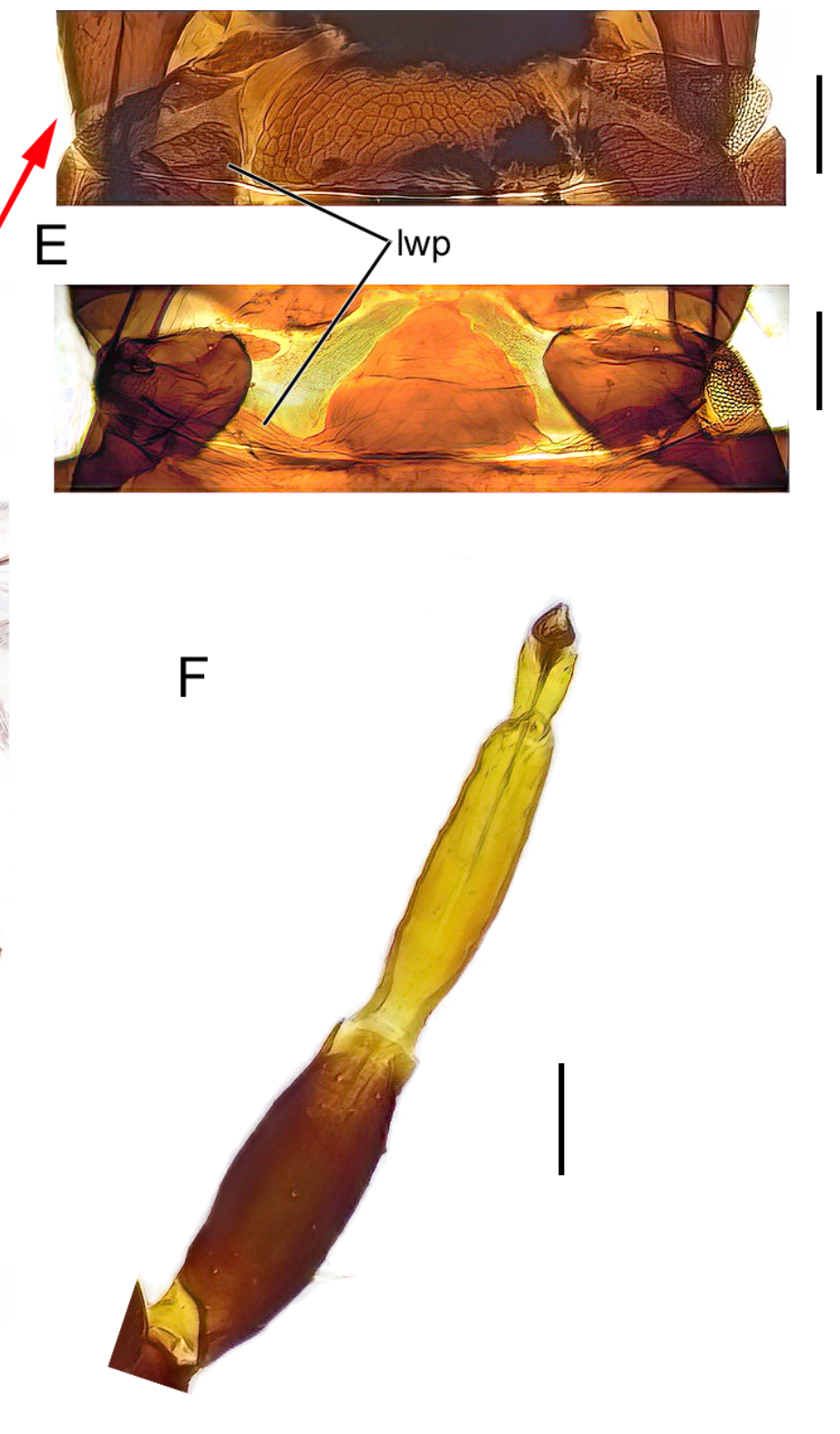

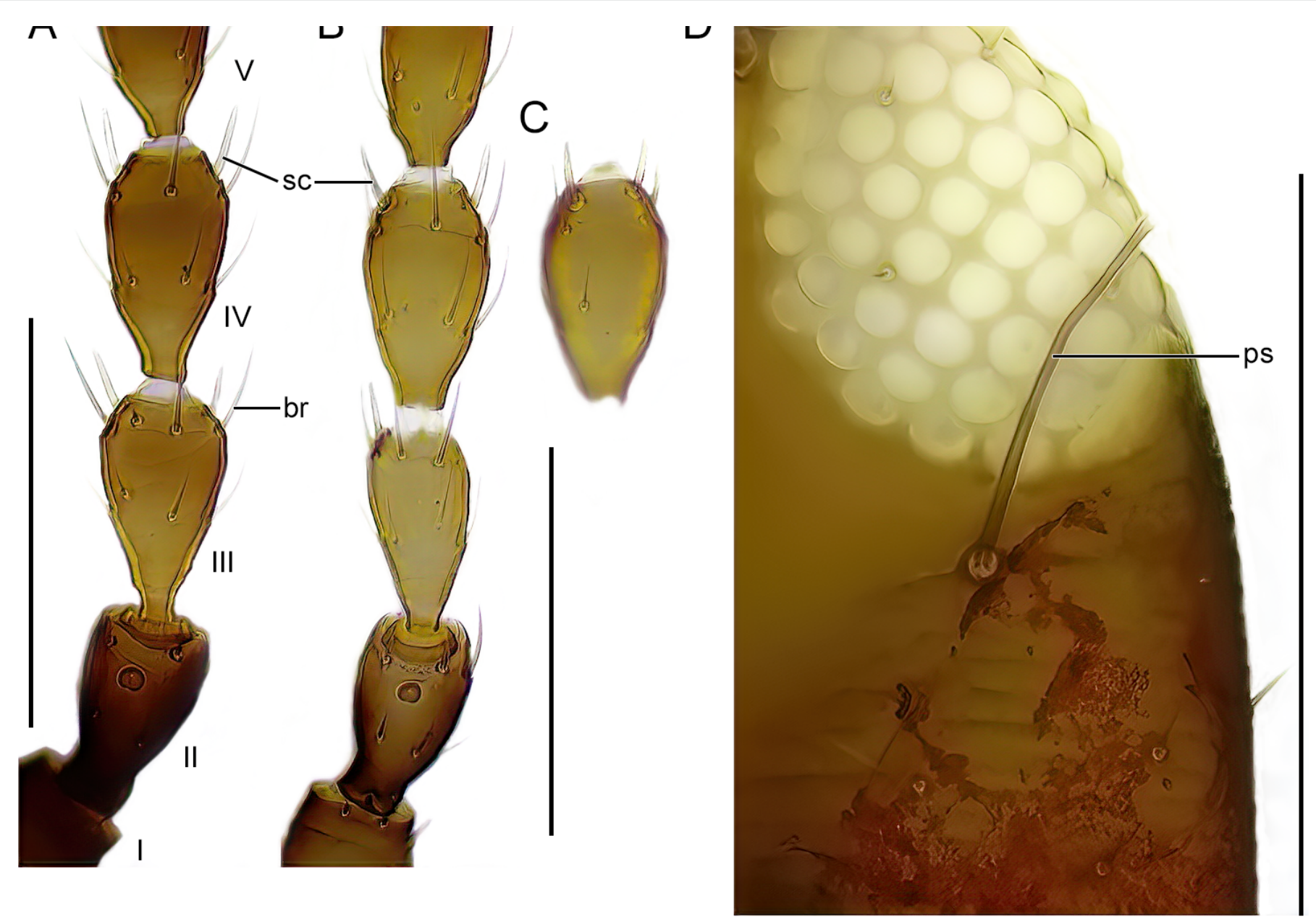

E

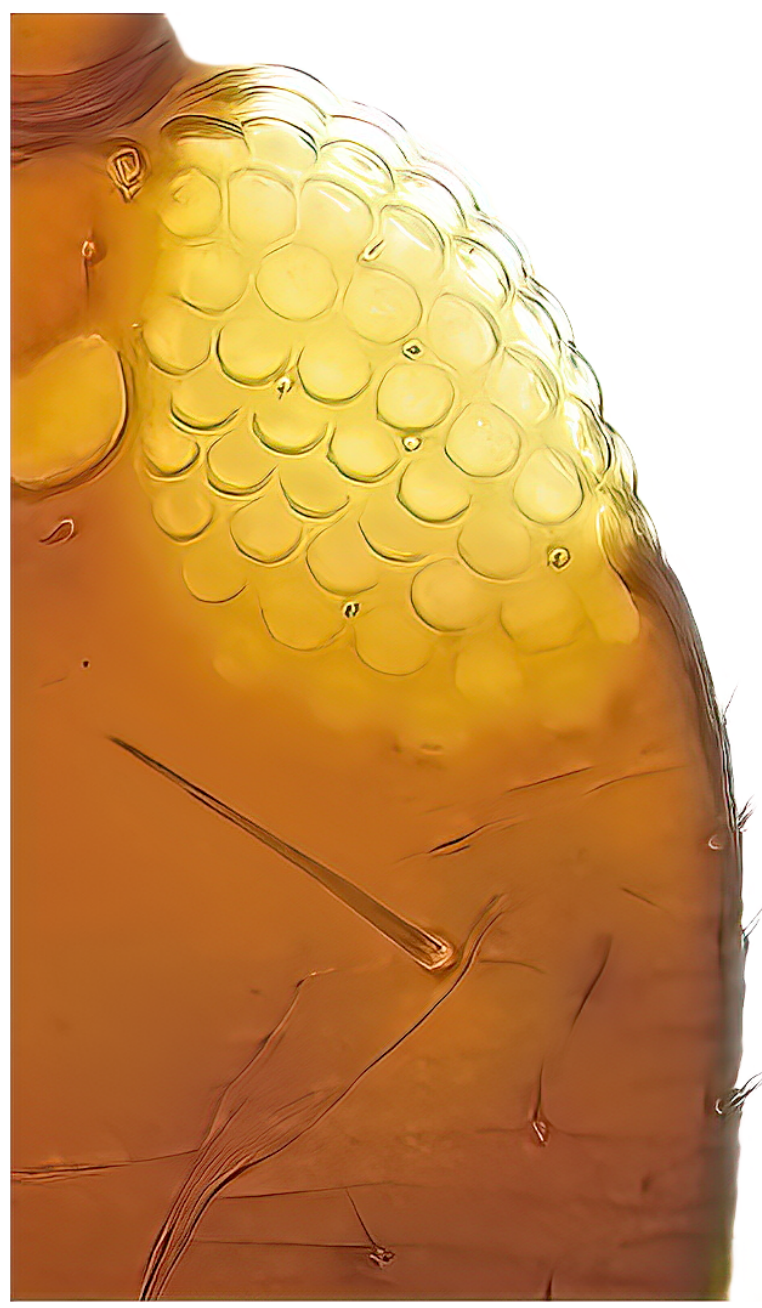

$\mathrm{F}$

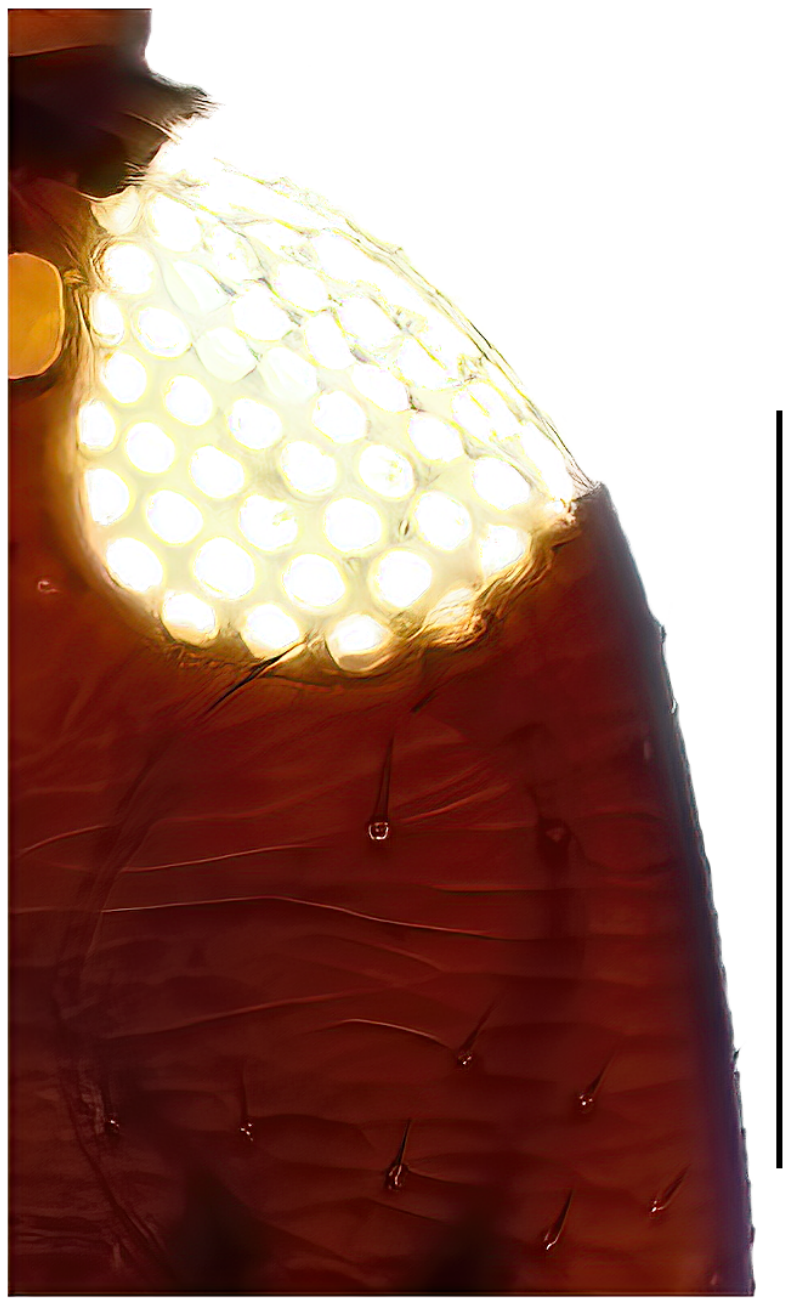



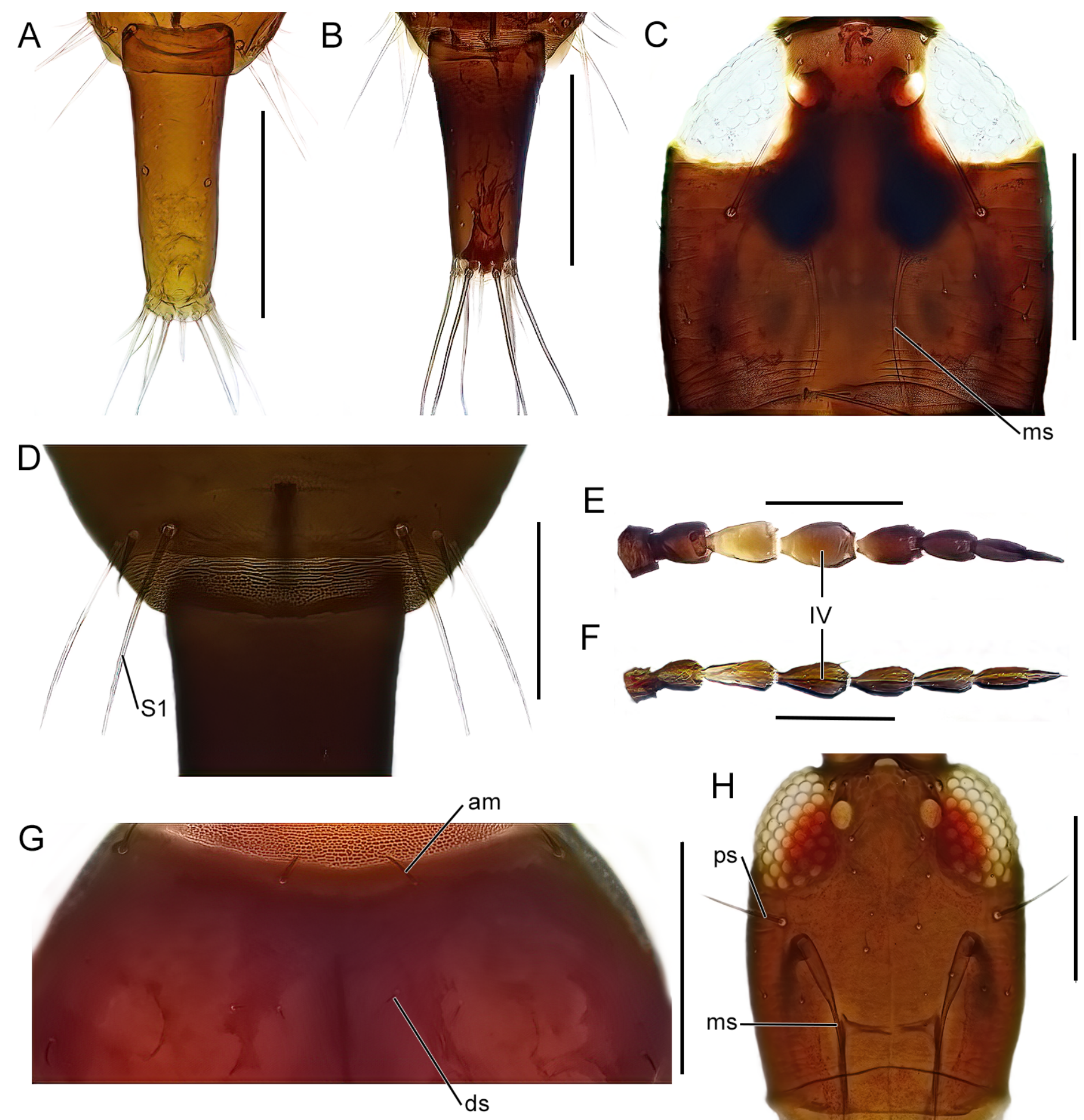

E
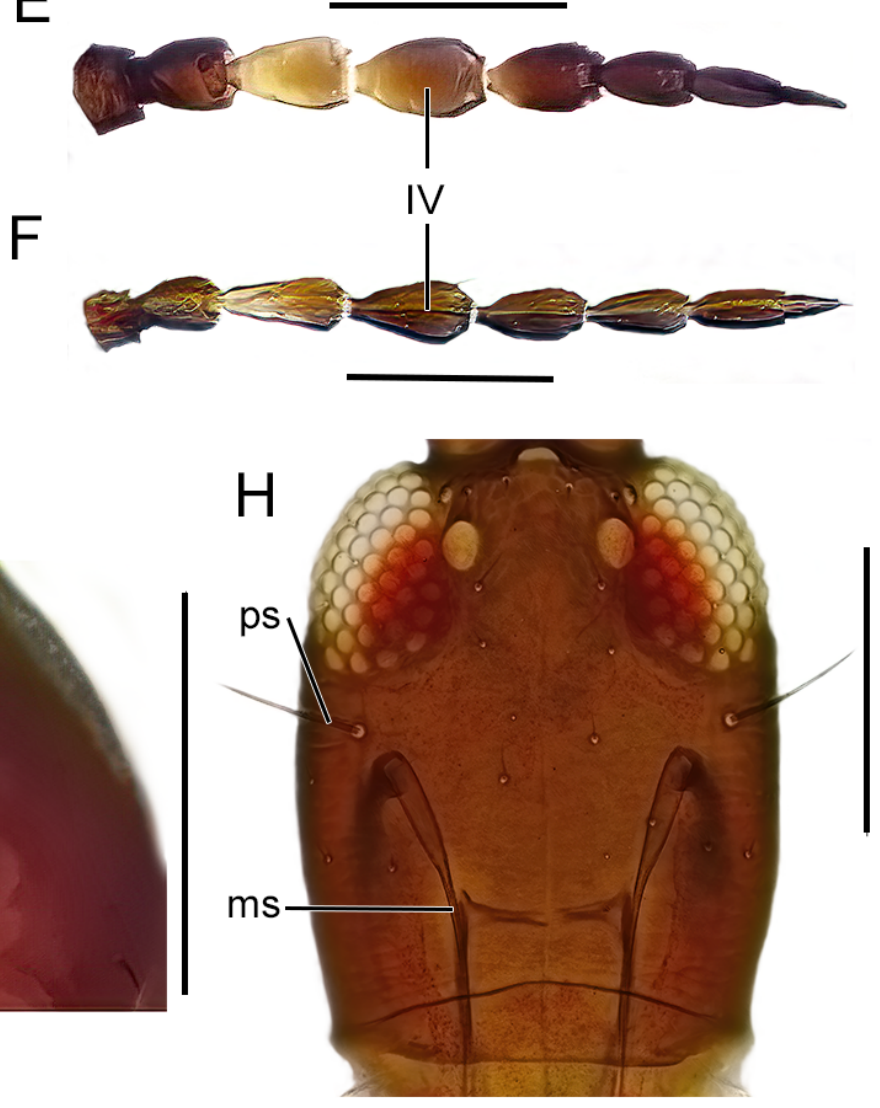

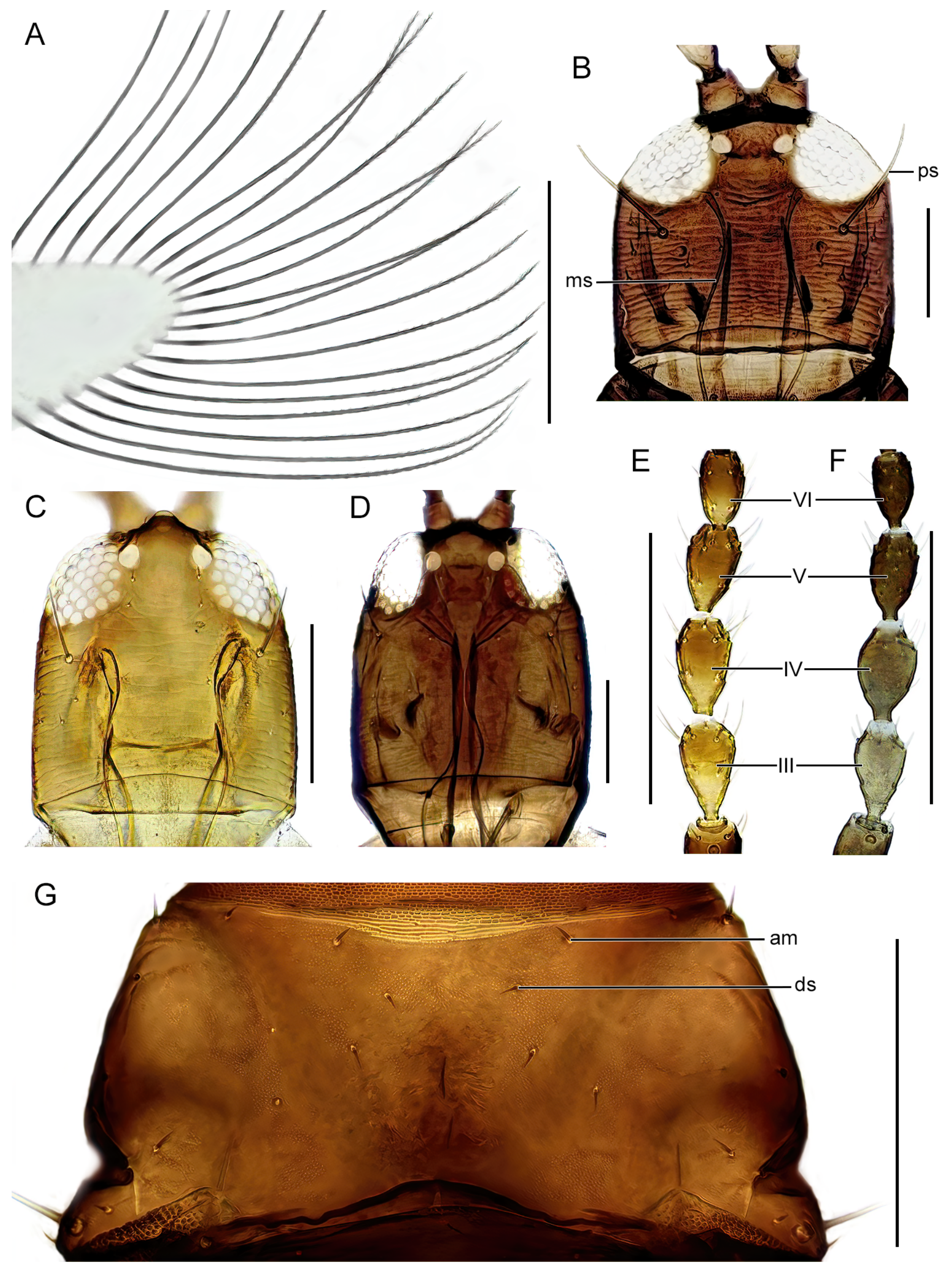


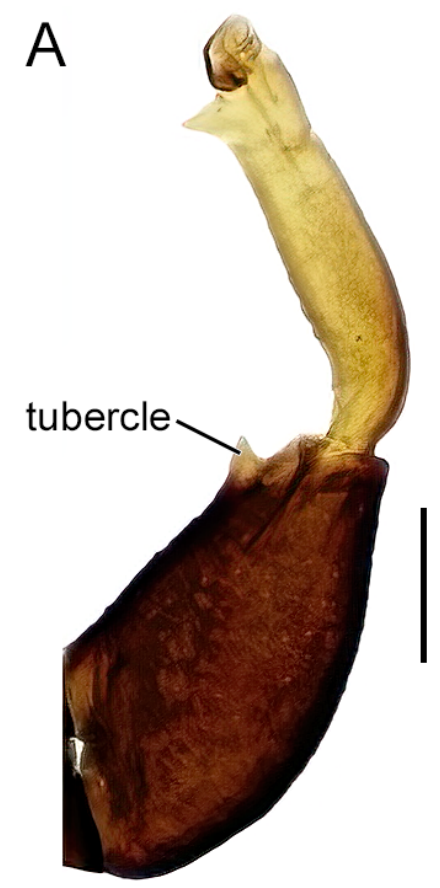

B

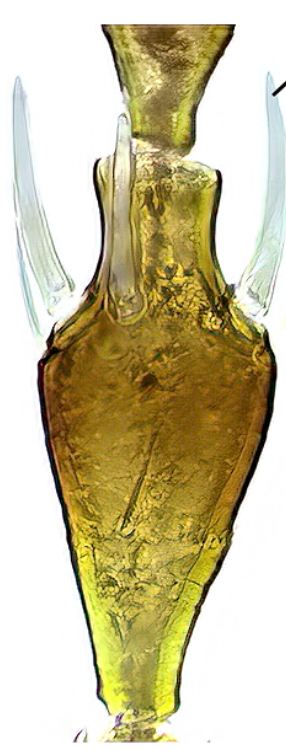

E

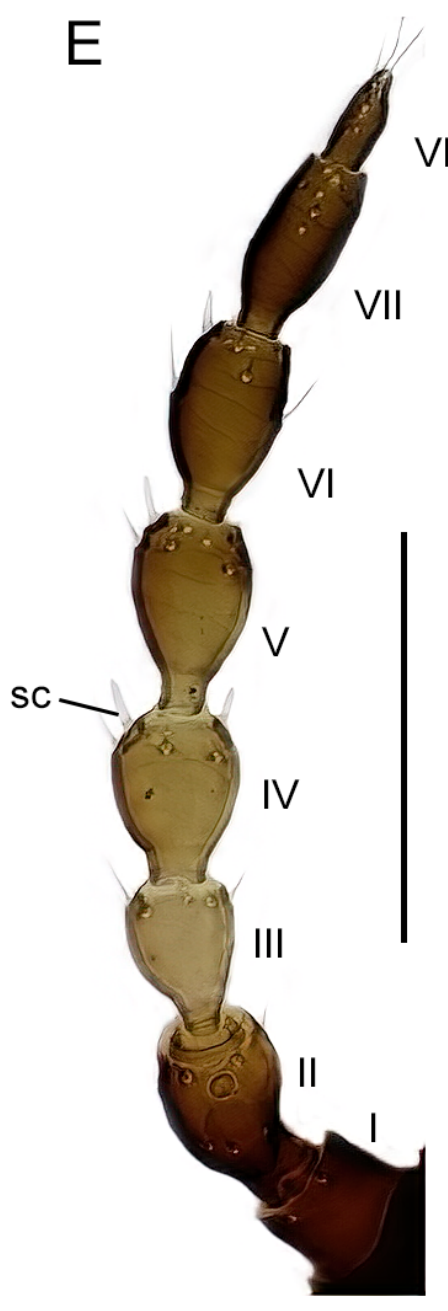

$\mathrm{F}$

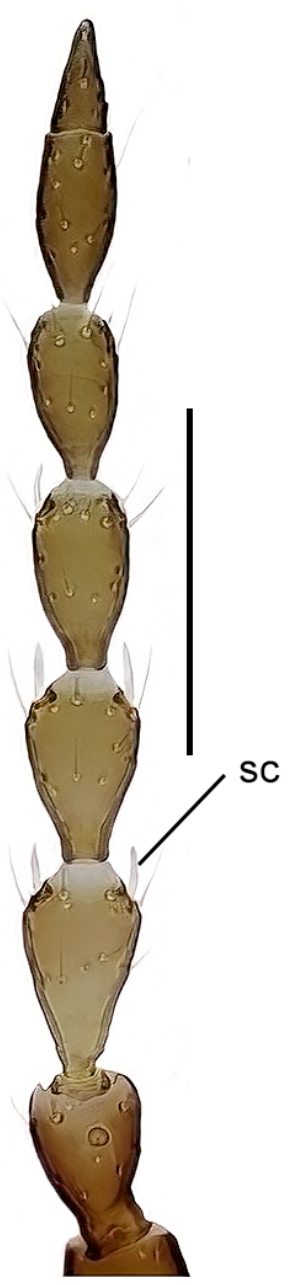

$\gamma^{\mathrm{sc}} \mathrm{C}$

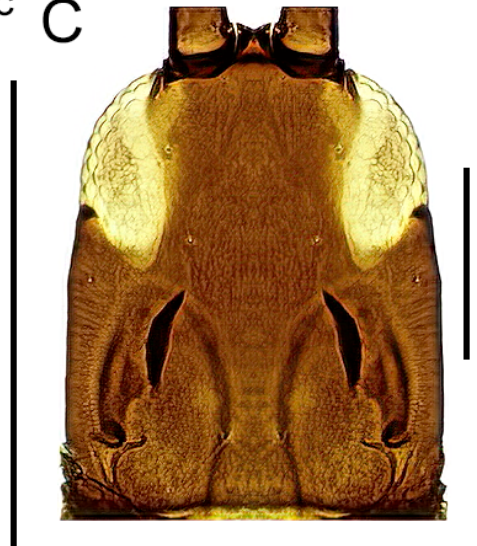

D

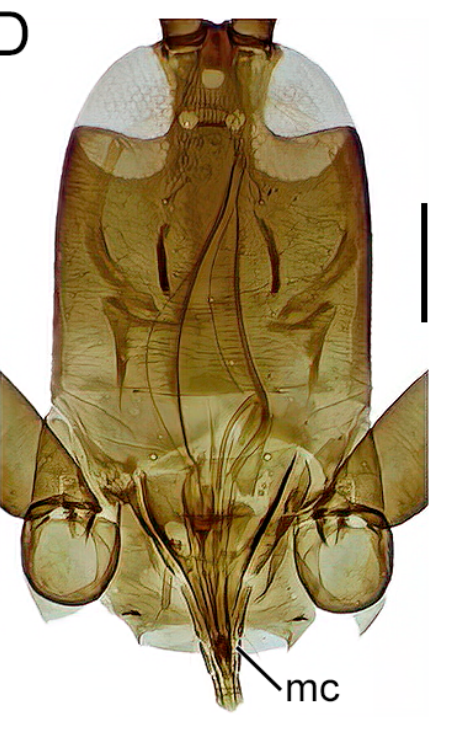

G

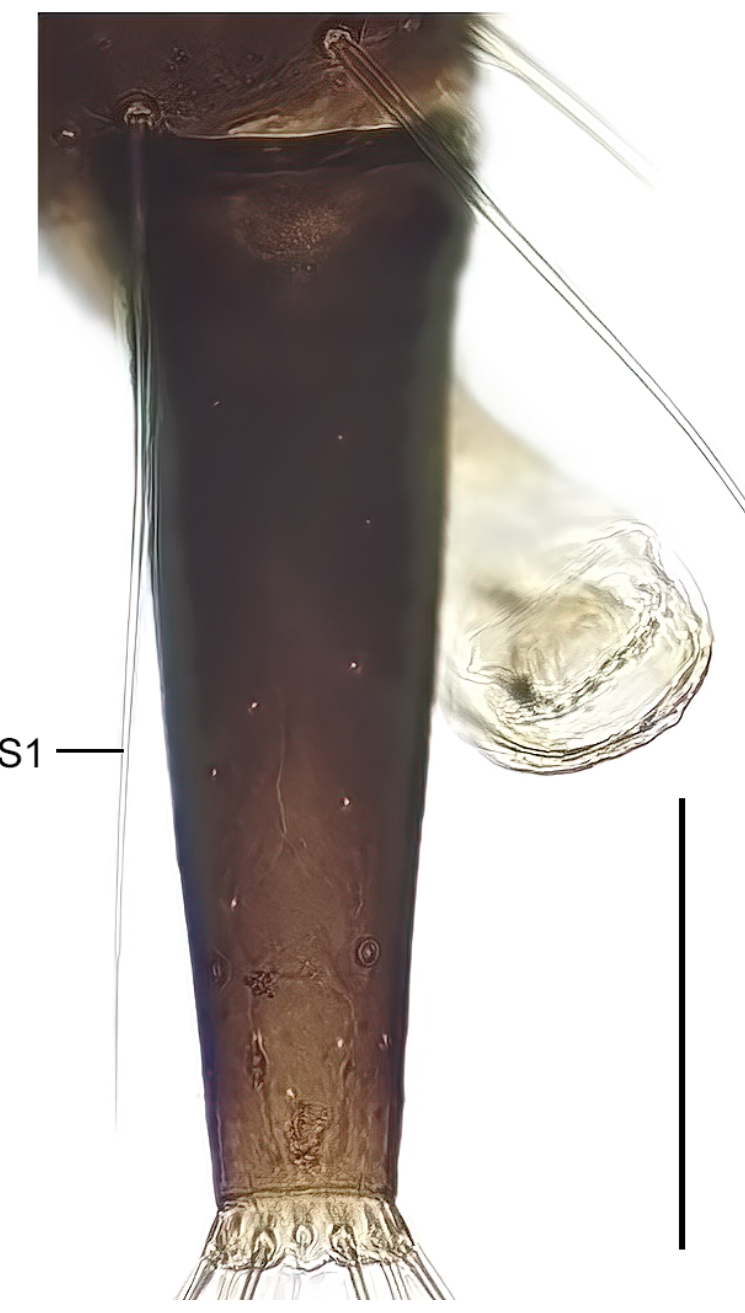



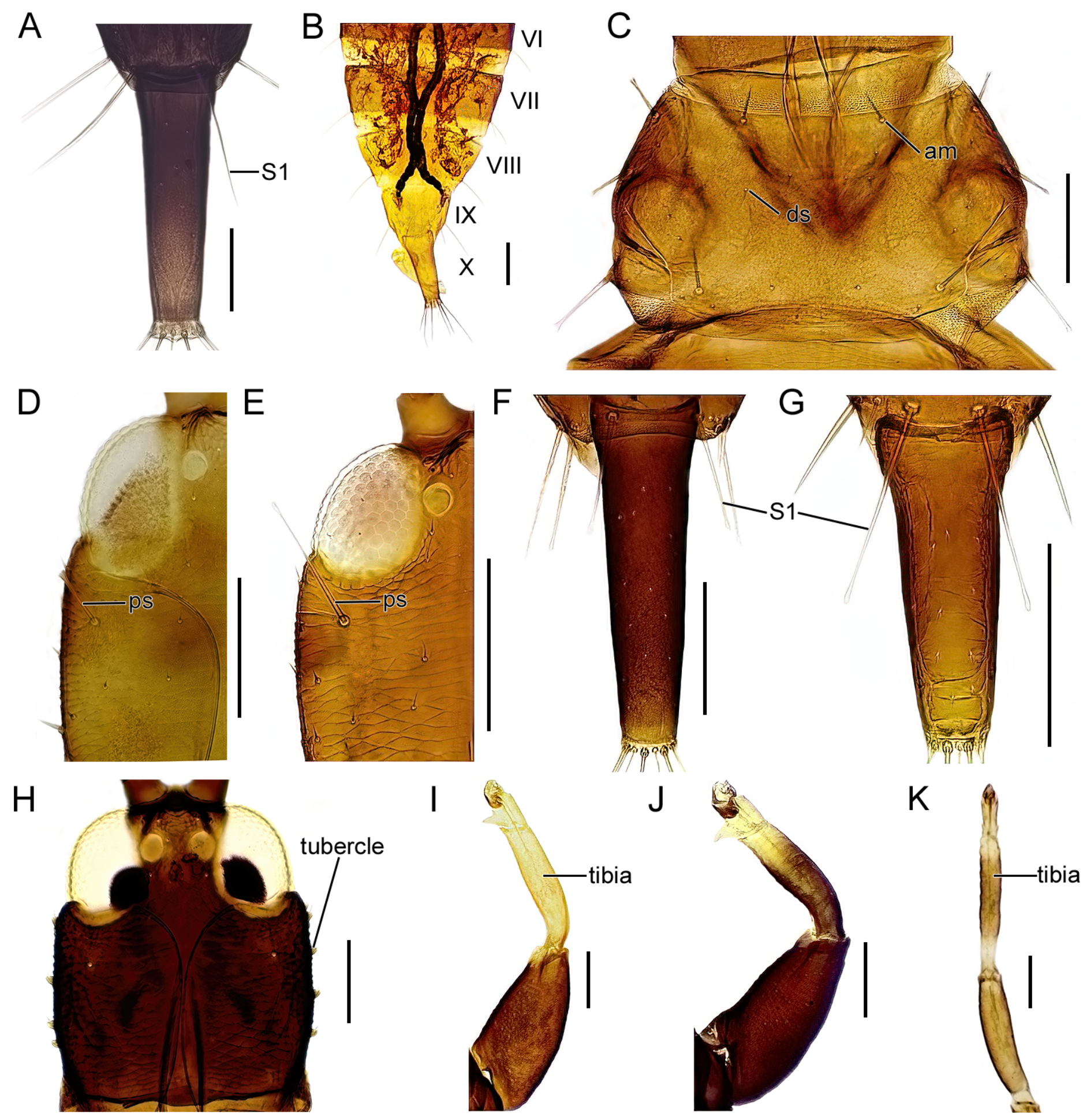


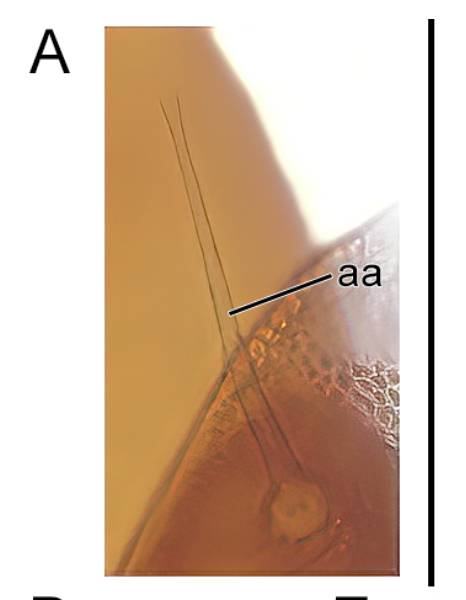

D
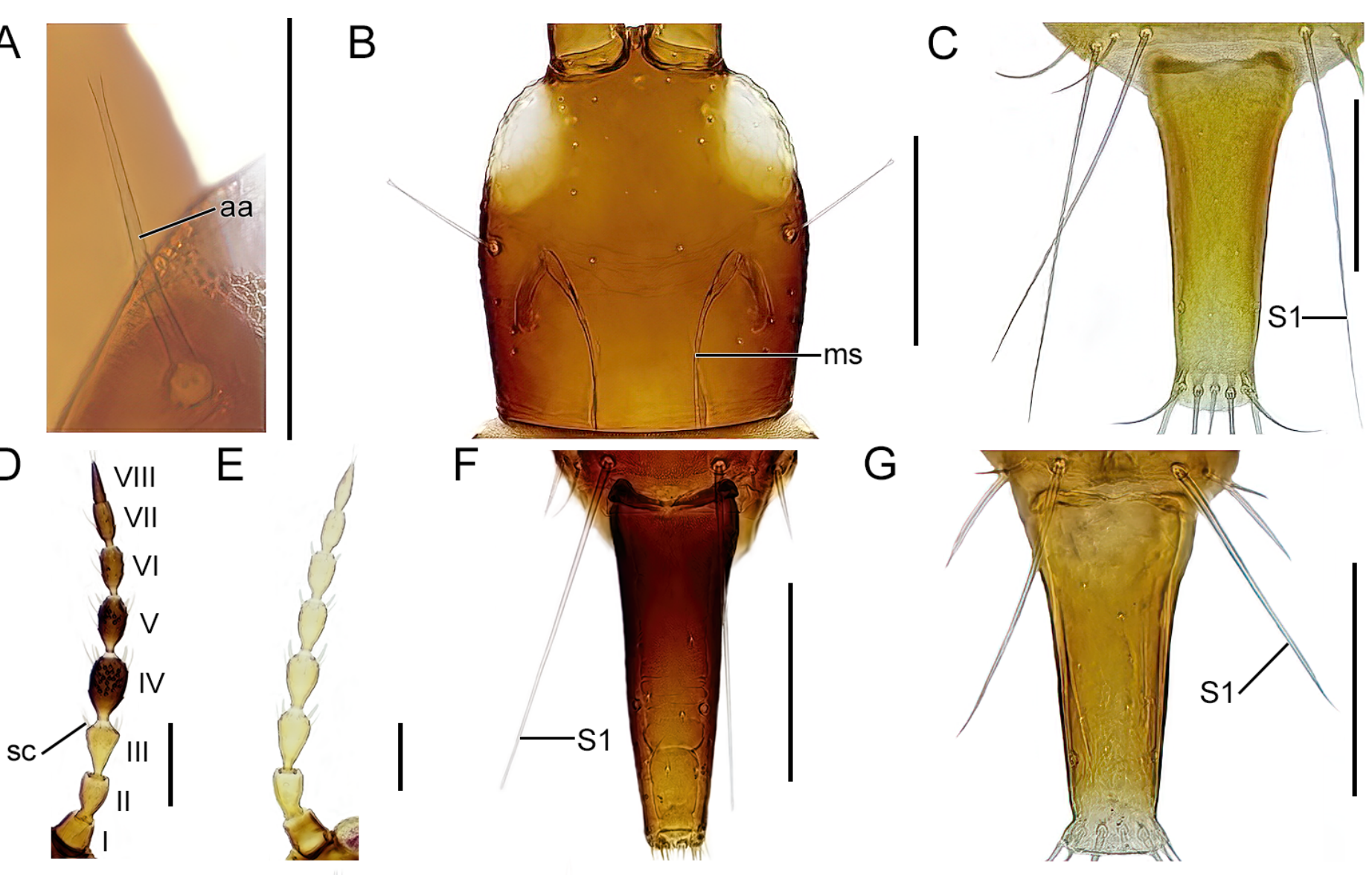

F

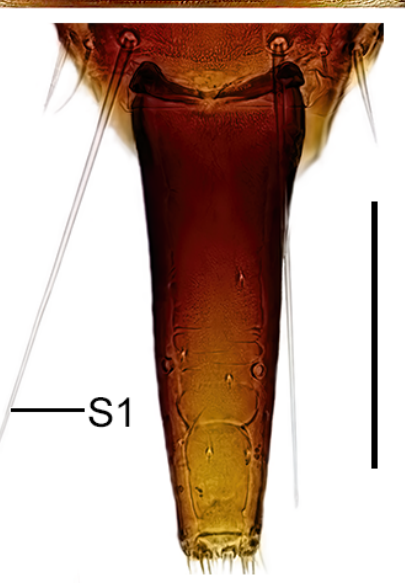

G
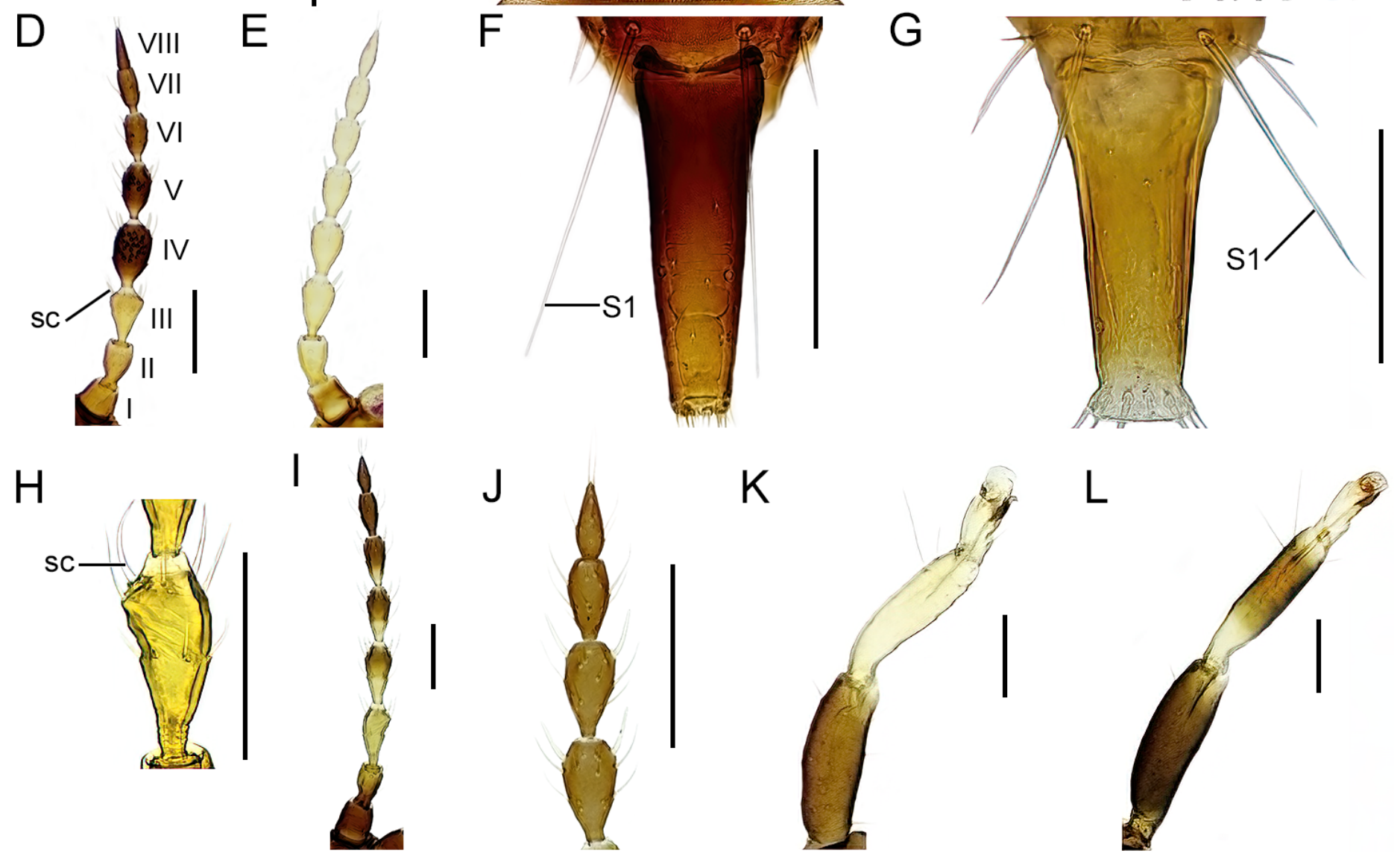